\title{
A compartmental CFD-PBM Model of High Shear Wet Granulation
}

Xi Yu ${ }^{1,2, *}$, Michael J. Hounslow ${ }^{2}$, Gavin K. Reynolds ${ }^{3}$, Anders Rasmuson ${ }^{4}$, Ingela Niklasson Björn $^{5}$, Per J. Abrahamsson ${ }^{4}$

1 European Bioenergy Research Institute (EBRI), School of Engineering and Applied Science, Aston University, Birmingham B4 7ET, UK

2 Department of Chemical and Biological Engineering, the University of Sheffield, Sheffield, S1 3JD, UK

3 Pharmaceutical Technology and Development, AstraZeneca, Macclesfield, SK10 2NA, UK 4 Department of Chemical and Biological Engineering, Chalmers University of Technology, SE-412 96 Gothenburg, Sweden

5 Pharmaceutical Technology and Development, AstraZeneca, Mölndal, SE-431 83 Mölndal, Sweden

*Corresponding author, email: x.yu3@aston.ac.uk

Keywords: High Shear Wet Granulation, Population Balance Model, Multiple Compartments, Monte Carlo, CFD

\begin{abstract}
The conventional, geometrically lumped description of the physical processes inside a high shear granulator is not reliable for process design and scale-up. In this study, a compartmental Population Balance Model (PBM) with spatial dependence is developed and validated in two lab-scale high shear granulation processes using a 1.9L MiPro granulator and 4L DIOSNA granulator. The compartmental structure is built using a heuristic approach based on computational fluid dynamics (CFD) analysis, which includes the overall flow pattern, velocity and solids concentration. The constant volume Monte Carlo approach is implemented to solve the multi-compartment population balance equations. Different spatial dependent mechanisms are included in the compartmental PBM to describe granule growth. It is concluded that for both cases (low and high liquid content), the adjustment of parameters (e.g. layering, coalescence and breakage rate) can provide a quantitative prediction of the granulation process.
\end{abstract}

\section{Introduction}

High shear granulation (HSG) is often applied in the chemical, agricultural, pharmaceutical, food, detergent and mineral industries to control a wide range of particle properties such as homogeneity, flowability, solid apparent density or particulate strength $^{1,2}$. Optimum 
granulation is capable of incorporating all primary particles into granules and gradually enlarging the granules to an intermediate size with a relatively narrow size distribution. HSG generally has a short processing time and consumes comparatively less liquid binder than fluidized bed or low shear mixer granulators ${ }^{3}$. Furthermore, HSG can handle viscous binders and produce small $(<2 \mathrm{~mm})$ granules with high density compared to other granulators $2,4,5$. Recent experimental investigations ${ }^{6,7,8}$ and numerical investigations ${ }^{9,10,11}$ of HSG reveal the advantage of this granulator both at particle scale and granulator scale. The mechanism of granulation (e.g. nucleation, growth and breakage) in wet granulation processes under mechanical mixing has been reviewed by Iveson et al ${ }^{12}$.

Population Balance Modelling (PBM) of HSG has been developed from a single dimensional model ${ }^{13,14}$ to a multi-dimensional model ${ }^{15,16,17,18,19,20,21,22}$. A review of the current PBM of HSG is shown in Table 1; the scope of this survey covers four aspects including description of granule properties, numerical solution technique, mechanisms and parameters estimation approach. As the understanding of granulation improves, the modelling strategy of HSG is developing from one-dimensional PBM with a single mechanism into multi-dimensional PBM with multiple concurrent mechanisms. Due to the complexity of the models, the solution technique of PBM has expanded from a purely deterministic approach to the coexistence of deterministic and stochastic. 
Table 1. Summary of recent literatures on PBM modelling of HSG




As a macroscopic predictive model, most PBMs are based on a well-mixed assumption, without spatial dependence. However, in a real HSG process, granulation rates (e.g. coalescence and breakage) are strongly dependent on the spatial position owing to heterogeneous mixing features of complex particulate flow. The theoretical models to predict the likelihood of granule coalescence in a granulator always require knowledge of the impact velocity, which has a wide distribution dependent on their positions and distances from the impeller. Furthermore, there is an upper velocity limit beyond which collisions result in granule breakage. Owing to such a distribution of impact velocity, breakage rates within the device is not a constant. Most granulation kernels used in PBM represent this distribution by an average value for the whole batch. Therefore, there is a need to develop a PBM with spatial dependence. Furthermore, due to the heterogeneous distribution of transformation kinetics and transport phenomena in particulate systems, the underlying mesoscopic models are either unknown or uncertain ${ }^{25}$. This scale gap becomes a hurdle to rational prediction of macro-scale performance directly from micro-scale simulations.

Compartmental modelling is a well-known technique which is frequently used for standard reactor engineering problems, and has been applied within crystallization for a number of years $^{26}$. Since the compartmental model allows a natural separation of kinetics and hydrodynamic mechanisms, it could make it possible to quantify kinetics and transport phenomena at the meso-scale. A reliable mesoscopic model could facilitate the development of a universal macro-scale model (e.g. PBM) to fill the scale gap in the granulation modelling platform. Furthermore, the compartmental model can deliver an accurate prediction for the distribution of product properties and provide reliable information for the optimization of design and operation.

Compartmental models using computational fluid dynamics (CFD) have been successfully applied to continuous steady-state evaporative crystallizers ${ }^{27,28,29}$, reactive semi-continuous and continuous precipitation processes ${ }^{30}$ and gas-liquid precipitation processes ${ }^{31}$.

In modelling of crystallization process, the motivation behand a compartmental model is the non-linear dependency of most physical processes (e.g. nucleation, growth, agglomeration and attrition) on the degree of super saturation, the energy dissipation, the crystal size and its distribution ${ }^{27}$. The hydrodynamics in a crystallizer vessel determine the non-uniform distribution of these process variables. The conventional, geometrically lumped description of the physical processes inside a crystallizer has, therefore, never proven to be reliable for scale-up purpose. 
Granulation is the process of formation of solid granule transformed from a powder bed with surrounding fluid (gas and liquid). The non-uniformity of mixing is even more extreme compared to the crystallization process. Analogously to crystallization, there is non-linear dependency of most physical processes on the velocity, particle (granule) properties (such as size and liquid content) and their distribution. Therefore, a similar modelling strategy to handle non-linear dependency of most physical processes on heterogeneity distribution of these process variables caused by imperfect mixing can be adopted based on the successful application of a compartmental model in crystallization.

\section{Objectives}

Due to the similarity between the two solid-based engineering systems, in this study we aim to develop a compartmental PBM model for HSG process with the aid of CFD model (i) to build a novel hybrid predictive model with spatial dependence (coupling hydrodynamics at meso-scale). (ii) to simulate the dynamic process in HSG and predict granule properties and their distribution in final product

\section{Heuristic approach}

The compartmentalization methods are divided into a heuristic approach ${ }^{27,32}$ and algorithmbased automatic zoning method ${ }^{33}$. The main advantages of automatic zoning approaches are: 1) a quick and reliable definition of a network of zones; 2) the possibility of increasing the model resolution (i.e. the number of zones) without major time expenditure during the model definition; 3) the ease of re-defining and adjusting previous zone models. However, the automatic zoning method can create a great number of very small zones with irregularly shaped boundaries, which makes the estimation of flow rate between interconnecting compartments complicated. Therefore, in this study, a heuristic approach is used to compartmentalize a high shear granulator aiding the development of a hybrid predictive compartmental model. By analogy with a multi-compartment crystallizer $^{32}$, the compartmental structure of a high shear granulator is based on hydrodynamic information obtained from CFD simulations including the overall flow pattern, velocity field and solids concentration field.

The product composition of granules is determined during their formation process. Thus product compositions are therefore strongly controlled by granulation mechanisms including wetting, nucleation, consolidation, coalescence, attrition and breakage occurring at particle 
scale $^{12}$. The process variables needed to be considered in the CFD simulation can be determined by the above correlation analysis in Table 2 . 
Table 2: Dependencies of granulation mechanisms on material composition, operation condition, geometry and hydrodynamic information

\begin{tabular}{|c|c|c|c|c|}
\hline Mechanisms & Material composition & $\begin{array}{l}\text { Operation } \\
\text { condition }\end{array}$ & $\begin{array}{l}\text { Equipment } \\
\text { geometry }\end{array}$ & $\begin{array}{l}\text { Solid-based } \\
\text { hydrodynamic }\end{array}$ \\
\hline $\begin{array}{l}\text { Nucleation } \\
\text { and wetting }\end{array}$ & $\begin{array}{l}\text { Contact angle, spreading coefficients, surface } \\
\text { tension, droplet size, granule size, granule porosity }\end{array}$ & $\begin{array}{l}\text { Spray rate and } \\
\text { impeller speed }\end{array}$ & $\begin{array}{l}\text { Nozzle position and } \\
\text { spray angle }\end{array}$ & $\begin{array}{l}\text { Bed moving } \\
\text { velocity }\end{array}$ \\
\hline Consolidation & $\begin{array}{l}\text { Granule porosity, binder viscosity, binder content, } \\
\text { granule size, surface tension }\end{array}$ & Impeller speed & Equipment type & $\begin{array}{l}\text { Impact velocity } \\
\text { (particle-wall) }\end{array}$ \\
\hline Coalescence & $\begin{array}{c}\text { Granule size, binder content, binder viscosity, } \\
\text { surface tension }\end{array}$ & $\begin{array}{l}\text { Impeller and } \\
\text { chopper speed }\end{array}$ & $\begin{array}{l}\text { Impeller and } \\
\text { chopper type }\end{array}$ & $\begin{array}{l}\text { Collision velocity } \\
\text { (particle-particle) }\end{array}$ \\
\hline $\begin{array}{l}\text { Attrition and } \\
\text { breakage }\end{array}$ & $\begin{array}{c}\text { Granule porosity, granule size, binder viscosity, } \\
\text { surface tension }\end{array}$ & $\begin{array}{l}\text { Impeller and } \\
\text { chopper speed }\end{array}$ & $\begin{array}{l}\text { Impeller and } \\
\text { chopper type }\end{array}$ & $\begin{array}{l}\text { Impact velocity } \\
\text { (particle-wall) }\end{array}$ \\
\hline
\end{tabular}


Four steps of a heuristic approach can be distinguished in the derivation of a compartmental model for a high shear granulator.

Firstly, primary compartments are selected to represent the overall flow pattern within the granulator. This is done on the basis of granulator dimension, geometry and operation condition. Two specific compartments, spray zone and chopper zone, are not built in CFD modelling since binder dispersion and the high impact region around the chopper are difficult to characterize in two fluid models. The spray zone is located beneath the nozzle and on the powder bed surface, where the liquid binder and powder surface first come into contact and form the initial nuclei. The approach to quantify the spray zone as a well-mixed compartment is examined by $\mathrm{Yu}$ et al ${ }^{34,35}$. The chopper zone is a high shear region where wet granules break due to impact, and can be used to limit the maximum granule size or to help distribute a viscous binder. The compartment size, location and flow rate can be estimated roughly by taking into account chopper type and its speed.

Secondly, the compartments are checked for gradients in granule velocity. Strong gradients may be expected around the impeller. If they are indeed present, one or more of the compartments selected in the first step will be split up.

Thirdly, all compartments are checked for the presence of solid concentration gradient, or in other words, the degree of solid phase mixing is analysed. This may result in further compartment subdivision.

Finally, the transportation rates at the boundary connecting the various compartments are determined.

\section{HSG systems}

Two lab-scale high shear granulators (case I, 1.9L MiPro granulator; case II, 4L DIOSNA granulator) are modelled and simulated in this study, because they have different liquid/powder ratio and flow pattern.

\section{Case I: 1.9L MiPro granulator}

In case I, the granulation of microcrystalline cellulose (MCC) mixture was performed by Björn et al ${ }^{36}$ in a $1.9 \mathrm{~L}$ lab-scale high shear granulator (MiPro, Procept, Belgium) with an inner diameter of $0.15 \mathrm{~m}$ and a three bladed bevelled impeller operating at 450rpm. Its schematic is shown in Fig. 1a. The total mass of solid was $280 \mathrm{~g}$. The mass of binder sprayed 
into the granulator was $14 \mathrm{~g}$ and the liquid addition period lasted for $2.5 \mathrm{mins}$. The amount of binding liquid (PVP K30) was set to $0.05 \mathrm{~g}$ liquid/g powder. Samples were withdrawn during the process after several seconds. Bulk density and NIR spectra were measured on the wet mass for each trial. Particle size distributions were measured on the material using image analysis (BeadCheck 830, PharmaVision Systems, Lund, Sweden)after drying in an oven at $50{ }^{0} \mathrm{C}^{36}$. These data allow the evolution of the liquid and solid phases to be measured (on a size-averaged basis). The operational parameters of case I are shown in Table 3.

Table 3: Operational parameters

\begin{tabular}{lll}
\hline Parameters & Case I & Case II \\
\hline Impeller speed [rpm] & 450 & 600 \\
Granulator diameter [m] & 0.15 & 0.225 \\
Granulator Height [m] & 0.11 & 0.113 \\
Primary particle density $\left[\mathrm{kg} / \mathrm{m}^{3}\right]$ & 1600 & 1600 \\
Solid loading [g] & 280 & 300 \\
Bowl size [L] & 1.9 & 4 \\
Addition time of liquid [min] & 2.5 & 4 \\
Wetting massing time [min] & 5 & 4 \\
Adding amount of liquid [g/g dry powder] & 0.05 & 0.42 \\
Binder density [kg/m ${ }^{3}$ ] & 1000 & 1000 \\
\hline
\end{tabular}

\section{Case II: 4L DIOSNA granulator}

In case II, the granulation of mixture (MCC, an active ingredient, and PVP) with water as liquid binder was performed by Darelius et al ${ }^{18}$ in a $4 \mathrm{~L}$ lab-scale high shear granulator (DIOSNA Dierks \& Soehne GmbH, Germany) with a three-bladed bevelled impeller rotating counter clockwise at $600 \mathrm{rpm}$ inside. Samples were withdrawn during the process after several seconds. The water content within granules was determined using loss on drying (LOD) (HR73 Halogen Moisture Analyzer, Mettler-Toledo AG). The particle size distribution was determined by sieving the dried granules into five size fractions. The schematic of $4 \mathrm{~L}$ DIOSNA granulator is shown in Fig. 1b. The operational parameters of case II are shown in Table 3.

\section{Flow regime}

The flow regimes of a powder bed of lactose in a 25L PMA fielder mixer granulator have been investigated by Litster et al ${ }^{37}$. The flow regime strongly depends on Froude number, which is defined as the ratio of rotational inertia to gravity. 


$$
F r=\frac{D N^{2}}{g}
$$

Litster et al ${ }^{37}$ found that bumping flow, where material rotates slowly and "bumps" as the impeller passes beneath, existed at $F r<1$; roping flow, where the bed rotates much faster and mixes vertically, existed at $F r>1$. The transition from bumping to roping is due to a change in the balance between the powder rotational inertia, which tends to push it towards the outside of the bowl, and gravity which tend to keep the bed surface horizontal. In this study, the flow pattern $(F r=0.86)$ for 1.9L MiPro granulator should be located in the bumping flow regime; the flow pattern $(F r=2.29)$ for $4 \mathrm{~L} D I O S N A$ granulator should be located in the roping flow regime, these flow regimes are validated in Fig. 5a and 14a.

\section{Multi-compartmental PBM}

In this study, a multi-compartmental PBM combines an Eulerian description of the granule internal properties (solid mass and liquid mass) and a Lagrangian description of granule external properties (spatial position). To account for spatial heterogeneity of mixing within processes for granule growth, a multi-compartmental PBM model (for compartment $i$ ) is develop as below

$$
\begin{aligned}
& \frac{V_{i} \partial n_{i}\left(m_{s}, m_{l}, t\right)}{\partial t}=V_{i} B_{0}^{0} B\left(m_{s}, m_{l}\right)+V_{i} R_{W}^{0} R_{W}\left(m_{s}, m_{l}\right)+V_{i} G_{0} \frac{\partial n_{i}\left(m_{s}, m_{l}, t\right) G\left(m_{s}, m_{l}, t\right)}{\partial m_{s}} \\
& +\frac{1}{2} V_{i} \beta_{0} \int_{0}^{m_{s}} \int_{0}^{m_{l}} \beta\left(m_{s}, m_{l}, m^{\prime}{ }_{s}, m^{\prime}{ }_{l}\right) n_{i}\left(m_{s}-m^{\prime}{ }_{s}, m_{l}-m^{\prime}{ }_{l}, t\right) n_{i}\left(m^{\prime}{ }_{s}, m^{\prime}{ }_{l}, t\right) d m_{s} d m_{l} \\
& -V_{i} \beta_{0} n\left(m_{s}, m_{l}, t\right) \int_{0}^{\infty} \int_{0}^{\infty} \beta\left(m_{s}, m_{l}, m^{\prime}{ }_{s}, m^{\prime}{ }_{l}\right) n_{i}\left(m^{\prime}{ }_{s}, m^{\prime}{ }_{l}, t\right) d m^{\prime}{ }_{s} d m^{\prime}{ }_{l} \\
& +V_{i} S_{0} \int_{m_{s}}^{\infty} \int_{m_{l}}^{\infty} S\left(m^{\prime}{ }_{s}, m^{\prime}{ }_{l}\right) b\left(m_{s}, m_{l}, m^{\prime}{ }_{s}, m^{\prime}{ }_{l}\right) n_{i}\left(m_{s}, m_{l}, t\right) d m^{\prime}{ }_{s} d m^{\prime}{ }_{l} \\
& -V_{i} S_{0} S\left(m_{s}, m_{l}\right) n_{i}\left(m_{s}, m_{l}, t\right)+\sum_{j=1, j \neq i}^{4} \frac{Q_{i \rightarrow j}}{V_{i}} n_{i}\left(m_{s}, m_{l}, t\right) \\
& +\sum_{j=1, j \neq i}^{4} \frac{Q_{j \rightarrow i}}{V_{j}} n_{j}\left(m_{s}, m_{l}, t\right)
\end{aligned}
$$

$m_{S}, m_{l}$ are the mass of solid and liquid contained in a granule; $n$ is a two dimensional number density function; $G_{0}, G$ are the layering rate constant and layering rate; $B_{0}^{0}, B$ are the nucleation rate constant and droplet density function for nucleation; $R_{W}^{0}, R_{W}$ are the rewetting rate constant and droplet density function for rewetting; $\beta_{0}, \beta$ are the coalescence rate 
constant and coalescence kernel; $S_{0}, S$ are the breakage rate constant and breakage kernel; $Q$ is volume flow rate; $V$ is the compartment volume. The high shear granulator is divided into five compartments using a heuristic approach based on CFD in this study. Since the chopper in both the CFD simulation and experiment is not used, four compartments are modeled.

\section{Transport rate}

In the CFD simulation, we assumed that the air and solid phases are incompressible. Therefore, the mass balance equation for both phases within compartments (e.g. 2, 3 and 4 in this study) of the granulator is

$$
\sum_{i=2}^{4}\left(\rho_{s} \bar{\varepsilon}_{i, s, t}+\rho_{a} \bar{\varepsilon}_{i, a, t}\right) V_{i}=\left(\rho_{s} \varepsilon_{s, 0}+\rho_{a} \varepsilon_{a, 0}\right) V
$$

For the particulate phase

$$
\sum_{i=2}^{4}\left(\bar{\varepsilon}_{i, s, t}\right) V_{i}=\varepsilon_{s, 0} V=\text { constant }
$$

For the particulate phase at time $t+\Delta t$ in compartment $i$ :

$$
\bar{\varepsilon}_{i, s, t+\Delta t} V_{i}=\bar{\varepsilon}_{i, s, t} V_{i}+\Delta t \sum_{j=2, j \neq i}^{4}\left(\Delta Q_{i \rightarrow j}+\Delta Q_{j \rightarrow i}\right)
$$

For the particulate phase at time $t+\Delta t$ in the granulator

$$
\sum_{i=2}^{4} \bar{\varepsilon}_{i, s, t+\Delta t} V_{i}-\sum_{i=2}^{4}\left(\bar{\varepsilon}_{i, s, t}\right) V_{i}=\Delta t \sum_{i=2}^{4} \sum_{j=2, j \neq i}^{4}\left(\Delta Q_{i \rightarrow j}+\Delta Q_{j \rightarrow i}\right)=0
$$

The numerical volume flow rates are estimated as

$$
\begin{aligned}
& \Delta Q_{j \rightarrow i}=\int_{\widetilde{u} \cdot d \tilde{S}<0} \varepsilon_{S} \cdot\left(\tilde{u}_{s}-r \tilde{\omega}\right) \cdot d \tilde{S} \\
& \Delta Q_{i \rightarrow j}=\int_{\widetilde{u} \cdot d \tilde{S}>0} \varepsilon_{S} \cdot\left(\tilde{u}_{s}-r \widetilde{\omega}\right) \cdot d \tilde{S}
\end{aligned}
$$

$\rho_{s}, \rho_{a}$ are the density of solid and air; $\bar{\varepsilon}_{i, s, t}, \bar{\varepsilon}_{i, a, t}$ are the volume fraction of particulate phase and gas phase in compartment $i$ at time $t ; V$ is the volume of whole granulator. $\varepsilon_{s, 0}, \varepsilon_{a, 0}$ are 
the volume fractions of particulate phase and gas phase at time $0 ; \Delta t$ is the time step determined by granulation rate; $V_{i}, V_{j}$ are the volume of compartment $i$ and $j ; \tilde{u}_{s}$ is the instantaneous velocity at the boundary between two compartments; $\widetilde{S}$ is the boundary area between two compartments; $\Delta Q_{j \rightarrow i}, \Delta Q_{i \rightarrow j}$ are the volume flow rates for particle phase between compartment $i$ and $j ; r$ is radius, $\widetilde{\omega}$ is angular velocity of rotating compartment 2 and 3.

\section{Algorithm for multi-compartment Monte Carlo simulation}

In this study, a constant volume Monte Carlo (CVMC) method ${ }^{38,39,40}$ is used to solve the PBEs in Eq.2. The CVMC is capable of solving multi-dimensional PBEs with both internal and external coordinates, and it can be easily extended to add additional informative coordinates of granules. To perform a simulation, the MMC (Multi-compartmental Monte Carlo) algorithm was written as outlined in Fig 2. The granule population of each compartment is symbolized as an array with $N_{P}$ rows to represent the granules and two columns for the internal coordinates. The shorthand $X_{j, k}$ refers to the $k$ th internal coordinate of the $j$ th particle (row $j$, column $k$ in the array). The granule population is updated at each time step, which is determined by granulation rate and estimated in Eq.14. During each time step, co-occurrence events (e.g. nucleation, rewetting, layering, coalesce and breakage) are implemented subsequently based on probability of events occurring at specific compartment. In this program, a dynamic allocation of array (DAA) ${ }^{40}$ algorithm is used to store and update the properties of particle population changing with time.

\section{Nucleation and re-wetting rates}

In this study, the dispersion of liquid binder starts and occurs in the spray zone, where two concurrent mechanisms, nucleation and rewetting controls the increase of liquid mass within granules. As the primary particles are smaller than the droplet, the kinetics of nucleation are described using an immersion mechanism and the operating regime corresponds with the droplet controlled regime in the nucleation map ${ }^{12}$. That is to say, one droplet forms a nucleus. The rewetting occurs when a droplet collides with an existing granule in the spray zone and the droplet is absorbed by the granule. These two mechanisms are directly related to the rate at which the liquid drops come into contact with the primary particles or the granules. Therefore, they depend on the addition rate of the liquid drops ${ }^{17}$. The initial expression of the drop addition rate is described by 


$$
r_{D}=\frac{v \dot{M}_{d r o p}}{V \bar{m}_{d r o p}}
$$

$r_{D}$ is independent drop addition rate; $\dot{M}_{d r o p}$ is spray rate; $\bar{m}_{d r o p}$ is mean mass of droplet; $v$ is volume of simulation box (sample size) in CVMC. The liquid flow rate and the drop mean mass can be determined from experimental data. Any drop entering the system can either produce a nucleus when contacting the primary particles, or rewet an existing granule. The probability of any of these two events happening is assumed to be approximately proportional to the projected surface area of each population. It follows as,

$$
\begin{aligned}
& b_{0}=\frac{A_{P}}{A_{P}+A_{G}} r_{D} \\
& r_{w}=\frac{A_{G}}{A_{P}+A_{G}} r_{D}
\end{aligned}
$$

with

$$
A_{P}=\frac{v c_{m, P}}{\bar{m}_{P}}\left(\frac{\bar{m}_{P}}{\rho_{P}}\right)^{2 / 3}, \quad A_{G}=\sum_{i=1}^{i=N_{G}}\left(\frac{m_{G, i}}{\rho_{G}}\right)^{2 / 3}
$$

A coarse estimate is made in Eq. 9c to save computational resources, because of the low sensitivity of the simulation to this parameter. $c_{m, P}$ is the mass concentration of primary particle in the simulation box; $\bar{m}_{P}$ is the mean mass of primary particles in the granulator; $A$ is a coarse estimate of the projected surface area; $m_{G, i}$ is the mass of granules in the simulation box; subscripts $G, P$ and $D$ represent granule, primary particle and droplet respectively. In the granulation process, different types and sizes of spray nozzle are used to transform the continuous liquid stream into disperse drops. The drop size distribution or average drop size is determined by the type and size of spray nozzle used in the experimental work. In principle, the experimental drop size distribution can be measured using laser diffraction (for example, the Spraytec (Malvern) ${ }^{17}$ ), however measurements were not reported in the experimental work and therefore, a plausible drop size distribution is used in the PBM, which is shown in Fig. 3. The geometric mean size of droplets is $94 \mu \mathrm{m}$. The geometric standard deviation is 1.67 .

\section{Coalescence and breakage rates}

The coalescence kernel to represent coalescence of particle $i$ and $j$ is defined as

$$
\beta_{i j}=\beta_{0} f\left(d_{i}, d_{j}\right) \psi\left(\Phi_{i}, \Phi_{j}\right)
$$

$d$ is the particle diameter; $\Phi$ is the liquid content; $i, j$ are indices related to two different particles. 
The coalescence rate constant, $\beta_{0}$, is representative of the process intensity. The size dependent part $f\left(d_{i}, d_{j}\right)$ characterizes the granule collision frequency and depends on the agitation model ${ }^{41}$. If a size-independent model is chosen, $f$ is equal to 1 . The coalescence efficiency, $\psi\left(\Phi_{i}, \Phi_{j}\right)$, describes the probability for two particles to aggregate accounting for their liquid content ${ }^{15}$.

The rate for size-dependent coalescence in the simulation box is

$$
r_{a g g}=\frac{1}{2} \beta_{0}\left\langle f_{i j}, \psi_{i j}\right\rangle v C_{n}^{2}
$$

Where:

$$
\begin{gathered}
\left\langle f_{i j}, \psi_{i j}\right\rangle=\frac{\sum_{i}^{N_{G}} \sum_{j \neq i}^{N_{G}} f_{i j} \cdot \psi_{i j}}{N_{P}\left(N_{P}-1\right)} \\
\psi_{i j}=\begin{array}{l}
1 \text { if } \max \left(\Phi_{i}, \Phi_{j}\right) \gg \Phi_{C} \\
0 \text { if } \max \left(\Phi_{i}, \Phi_{j}\right)<\Phi_{C}
\end{array}
\end{gathered}
$$

A simple equipartition of kinetic energy (EKE) coalescence kernel is used to model the size dependent collision frequency in order to restrain the number of kinetic parameters and facilitate the interpretation of results. This expression is

$$
f_{i j}^{E K E}=\left(v_{i}^{1 / 3}+v_{j}^{1 / 3}\right)^{2} \sqrt{\frac{1}{v_{i}}+\frac{1}{v_{j}}}
$$

$v_{i}$ is particle volume

The breakage of wet granules will influence and may control the final size distribution, especially in high shear granulators. Granule breakage is a function of formulation properties and operating variables. Size-independent breakage is assumed, giving the following breakage rate:

$$
r_{\text {break }}=S_{0} v c_{n}
$$

$S_{0}$ is the breakage rate constant; $c_{n}$ is the total particle number per unit volume. In the sizeindependent breakage case, the probability of occurrence of breakage for each particle is the same and therefore the particle is randomly selected.

\section{Time step}

Due to the diverse range of granulation rates across all compartments, a constant time step, calculated in spray zone, is applied to all compartments over the simulation period. In the spray zone, the time interval is calculated from the addition rate of liquid binder for every $n_{D}$ 
$\left(n_{D}>1\right)$ droplets introduced, so that there is $n_{D}$ integer numbers of droplet-adding events per time interval. The time interval is

$$
\Delta t=\frac{n_{D}}{r_{D}}
$$

The number of granulation events (coalescence and breakage) per time interval in compartment 2 and 4 are:

$$
\begin{aligned}
n_{\text {coal }} & =\Delta t \times r_{\text {coal }} \\
n_{\text {break }} & =\Delta t \times r_{\text {break }}
\end{aligned}
$$

It is possible for $n_{\text {coal }}$ and $n_{\text {break }}$ to be non-integers. In this case, the fractional part can be easily implemented in the Monte Carlo simulation by choosing a random number.

\section{Layering}

Layering describes granule size growth and uptake of primary particles. It initiates immediately after the introduction of a drop into the powder bed containing sufficient primary particles. This mechanism is considered as a continuous process to increase solid mass within granule. A model has been developed on the basis of a diffusion phenomenon by Hounslow et al ${ }^{42}$. It assumes that the mass of primary particles incorporated into the granule is related to an effective diffusion rate at its surface. For a spherical granule, an approximate analytical solution of this problem is given as

$$
\frac{d m_{s, i}}{d t}=\frac{12 D_{e f f} \Phi_{c p}^{2 / 3}}{\left(\frac{3 m_{L, i}}{4 \pi \rho_{L}}\right)^{2 / 3}}\left(\frac{1-\Phi_{c p}}{\Phi_{c p}} \frac{\rho_{L}}{\rho_{S}} m_{L, i}-m_{S, i}\right)
$$

$D_{e f f}$ is binary effective diffusivity; $\Phi_{c p}$ is liquid content in the critical packing state; $\rho$ is density; subscripts $S$ and $L$ represent solid and liquid respectively; $i$ is index related to particles $i$. This expression is numerically integrated for each individual granule and between two adjacent time steps by a simple Euler's method to save computing time. The accuracy is normally sufficient because of the high frequency of the different processes and the very small time step. For a single nucleus formed from a single drop of liquid, layering might continue until the nucleus is essentially uniform in solid content. For a collection of particles, formed from a collection of drops, the process will continue until either all the nuclei are each of uniform content, or until there are no more free primary particles.

In this study, two lab-scale HSG cases are used to validate the model. Five mechanisms were included and parameterized as follows:

- The nucleation rate in the spray zone, $b_{0}$, was determined from the spray rate $r_{D}$ and 
the droplet size distribution (Eq. 9a)

- The rewetting rate was determined from Eq. 9b, using the spray rate

- The spatially-independent layering rate was determined using Eq. 16, by estimating $\Phi_{C P}$, which determines the maximum size a granule can achieve when it is saturated with powder, and $D_{e f f}$, which controls the overall layering rate.

- The coalescence rate in Bulk zone $4, \beta_{0}$, which controls growth by increasing granule size was estimated for particular cases

- The breakage rate in Impeller zone $2, S_{0}$, which controls growth by decreasing granule size was also estimated for particular cases.

\section{CFD Model}

In this study, we present a two fluid model (TFM), which is able to aid the compartmentalization of a high shear granulator and provide a numerical transport rate among different compartments. The gas-solid flow was investigated using TFM based on the Kinetic Theory of Granular Flow (KTGF). The model was solved using the CFD software ANSYS-FLUENT Version 12. Whilst for high shear granulation systems, the discrete element model (DEM) approach can provide predictive capabilities by resolving microscopic particle-particle and particle-wall interactions, 43, 44, 45, 46, 47, 48 it suffers from being computationally expensive, making it unattractive to handle realistic situations where a large number of particles are processed ${ }^{43}$.. The CFD method (continuum approach) provides an alternative option to simulate the bulk flow in high shear granulation, and recent developments of CFD models and evaluations of its validity on HSG system can be found in the literatures $49,50,11,51,52$. Darelius et al ${ }^{50}$ commented that the advantage of using the Eulerian-Eulerian approach (KTGF+ friction) compared to discrete element methods is that there is no computational limitation on the number of particles being modelled, and thus manufacturing scale granulators can be modelled as well. The CFD model (KTGF + friction) used in the study has been quantitatively validated using experimental data several times. Darelius et al ${ }^{50}$ validated the, KTGF+ friction, CFD model with experiment data. They found that the bed height could be well predicted by implementing the partial slip model, although there was some discrepancies in the velocity profile compared with experimental observations, for example, the swirling motion of the rotating torus formed was overpredicted and the tangential wall velocity was under-predicted. The validation of the KTGF + friction CFD model can also be found in the work of another research group, $\mathrm{Ng}$ et al, 
${ }^{49}$ concluded that the KTGF + friction, Eulerian based continuum model captures the main features of solids motion in high shear mixer granulator including the bed height and dominating flow direction (the tangential velocity). However, the continuum based kineticfrictional model is not capable of capturing the complex vertical swirl pattern. The CFD simulation of 1.9L MiPro granulator using KTGF and frictional stress models has been performed by Darelius et al ${ }^{50}$ and the identical mesh used in this study. The CFD model (Euler-Euler) assumed similar material properties for all the different components of the blend. To be able to compare the simulated results with experimental data, the simulated model powder was set with properties similar to coarse microcrystalline cellulose (mcc) with mono-dispersed particles of $59 \mu \mathrm{m}$ in diameter and $740 \mathrm{~kg} / \mathrm{m}^{3}$ in density. The sliding mesh technique is used and a total of 157,368 cells are built to resolve the $3 \mathrm{D}$ flow field. The sliding mesh approach is employed to tackle the difficulty of the rotation of the impeller blades. In case II, a total of 81,305 cells are built to resolve the 3D flow field. The impact of the grid size on the solution accuracy was initially tested by setting three different meshing schemes and the grid size used in this study was found to give acceptable grid independent solution.

\section{Computational procedure}

The model equations were solved using the finite volume approach. First-order discretization schemes were used for the solution of the convection terms in all governing equations. The relative error between any two successive iterations was specified by using a convergence criterion of $6.5 \times 10^{-4}$ for momentum equation and $1 \times 10^{-3}$ for other equations. The phasecoupled SIMPLE (PC-SIMPLE) algorithm ${ }^{53}$, which is an extension of the SIMPLE algorithm to multiphase flows, was applied for the pressure-velocity coupling. The linearized equations for governing equations were solved using a block algebraic multigrid method. In order to ensure easy convergence of the various partial differential equations (PDE) in the model, the Courant-Friedrichs-Lewy (CFL) condition for three-dimensional PDE is followed:

$$
C=\frac{u_{x} \Delta t}{\Delta x}+\frac{u_{y} \Delta t}{\Delta y}+\frac{u_{z} \Delta t}{\Delta z} \leq C_{\max }
$$

where $C_{\max }$ is specified by the CFL condition to fall within the range of $\sim 1-5^{54,55,56}$. In this study, a time step of 0.0007 seconds was found to satisfy this condition. The time step 0.0007 is corresponding to a $2.52^{\circ}$ impeller rotation in the TFM simulation of case II. One simulated revolution took approximately 6 hours on a PC with Duo CPU $3.00 \mathrm{GHZ}$ and 3GB of RAM. 


\section{Results and discussions}

\section{Case I}

\section{Overall flow pattern and velocity field}

The overall flow pattern produced from the CFD simulation demonstrates a swirling motion of the rotating torus within the high shear granulator using a three-bladed bevelled impeller as shown in Fig. 4. The highest velocities and their gradients occur in the impeller zone both in the radial and tangential direction. The flow in the bulk zone of the granulator has less steep gradients and a more reduced and uniform velocity when compared with the flow in the impeller zone. Very small velocity vectors are shown in the relatively large upper zone of the granulator since the air phase in TFM is present above the powder bed. In the bulk zone or 'inner core' of the granulator, axial flow in the downward direction back into the granulator impeller zone dominates.

The overall flow pattern is applied initially to construct the compartmental modelling framework for the high shear granulator. However, this is rather arbitrary and is subject to further refinement using the solids concentration profile. The high shear granulator is split into two compartments, (i) the impeller zone, which takes the high impact of particles into account, and (ii) the bulk zone, where lower velocities dominate in the upper region of the powder bed.

\section{Solid concentration}

Fig. 5 shows the solid concentration (volume fraction) distribution in the high shear granulator using a three-bladed bevelled impeller. The flow regime of bumping flow, where material rotates slowly and the powder bed surface "bumps" as the impeller passes beneath it is seen in Fig.5a. The solid is heterogeneously distributed throughout the granulator. A high concentration of solid particles appears under the impeller and around the shaft at the bottom of the granulator. This is similar to a dead zone beneath an impeller in a crystallizer ${ }^{32}$, due to reduced velocity and mixing intensity in this region producing sedimentary effects. This causes unwanted particle deposition and the formation of a high solid concentration zone. For a vertical axis bladed mixer, particles rise to form a heap in front of the blade then either flow downward on the bed surface over the blade or to the base of the heap to re-join the flow toward the blade. There is a recirculating zone in front of the blade ${ }^{57,58}$. This is due to a high solid concentration which only exists in the vicinity of the impeller blade. The impeller zone 
is further divided into two rotated compartments, impeller zone (Zone 2) and rotated bulk zone (Zone 3) in Fig. 5b and Fig 6b.

\section{Compartmentalization structure}

The compartmental structure (Fig. 6) for the HSG was derived from the granulator dimensions, geometry, operating condition, particle flow and solid concentration. The simulation of the compartmental model for HSG can be performed with five compartments: Spray zone 1, Impeller zone 2, Bulk zone 3 (rotated compartment), Bulk zone 4 and Chopper zone 5 .

The compartmental model is applicable to both case I and case II. The location of compartmental boundaries in case I is shown in Table 4.

Table 4: Location of compartmental boundaries

\begin{tabular}{ccccc}
\hline \multicolumn{5}{c}{ Location of boundary } \\
Type & Compartments & $\theta\left(^{\circ}\right)$ & $z(m)$ & $r(m)$ \\
\hline \multirow{2}{*}{ Case I: 1.9 L MiPro } & $2 \& 3$ & $0,40,120,160,240,280$ & $0-0.022$ & $0-0.075$ \\
granulator & $2 \& 4$ & $0-40,120-160,240-280$ & 0.022 & $0-0.075$ \\
& $3 \& 4$ & $40-120,160-240,280-360$ & 0.022 & $0-0.075$ \\
Case II: 4L DIOSNA & $2 \& 3$ & $0,40,120,160,240,280$ & $0-0.03$ & $0-0.1125$ \\
granulator & $2 \& 4$ & $0-40,120-160,240-280$ & 0.03 & $0-0.1125$ \\
& $3 \& 4$ & $40-120,160-240,280-360$ & 0.03 & $0-0.1125$ \\
\hline
\end{tabular}

In order to obtain reliable simulation results, a fully developed two-phase (solid and air) flow field has been built after running TFM simulations over a long period of flow time, which is equivalent to approximately one hundred rotations of the impeller. Then the temporal history of the numerical transport rate among multi-compartments are recorded using a User Defined Function (UDF) within ANSYS-FLUENT V12. The temporal history of the numerical transport rate is monitored over a sufficiently long period of flow time, which is equivalent to approximately sixteen rotations of the impeller. The sample number at the boundary surface (Eq. 7) between compartments is optimized for estimating transport rate. For example, in case II, as the number of sample points increases, the relative error (overall net transport rate based on mass balance) decreased (in Fig 7).

The temporal history of the volume flow rate and volume-based average solid volume fraction at each compartment is shown in Fig 8a and Fig 8b, respectively. The variation range is less than $25 \%$, therefore we applied a time-averaged volume flow rate (Table 5) to 
represent the transport rate among different compartments, which can provide hydrodynamic information for the macroscopic model (PBM).

In order to maintain mass conservation of the granule population and avoid undermining the integrity of the sample distribution, the same sample ratio, 1/500 of compartment volume is adopted in the Monte Carlo simulation in each compartment.

The transport rate is the time-average volume flow rate, which is estimated using CFD in part I of this study. Due to lack of experimental data for nozzle angle and height of nozzle, the size of the spray zone and its transport rate with adjacent compartment (e.g. Bulk zone 4) were estimated in Table 5 and 6.

Table 5: Transport rate

\begin{tabular}{ccc}
\hline Volume flow rate $\left[\mathrm{m}^{3} / \mathrm{s}\right]$ & Case I & Case II \\
\hline$Q_{14, m}$ & $9.053 \times 10^{-4}$ & $7.513 \times 10^{-3}$ \\
$Q_{41, m}$ & $9.053 \times 10^{-4}$ & $7.513 \times 10^{-3}$ \\
$Q_{23, m}$ & $6.879 \times 10^{-3}$ & $1.306 \times 10^{-2}$ \\
$Q_{32, m}$ & $1.025 \times 10^{-2}$ & $2.571 \times 10^{-2}$ \\
$Q_{34, m}$ & $4.368 \times 10^{-3}$ & $4.528 \times 10^{-3}$ \\
$Q_{43, m}$ & $1.907 \times 10^{-3}$ & $2.596 \times 10^{-3}$ \\
$Q_{24, m}$ & $1.130 \times 10^{-3}$ & $4.930 \times 10^{-3}$ \\
$Q_{42, m}$ & $8.413 \times 10^{-4}$ & $7.326 \times 10^{-4}$ \\
\hline
\end{tabular}

Table 6: Volume of each compartment and its sample size

\begin{tabular}{ccccc}
\hline & \multicolumn{2}{c}{ Case I } & \multicolumn{2}{c}{ Case II } \\
\hline Compartment & Volume $\left[\mathrm{m}^{3}\right]$ & Sample ratio & Volume $\left[\mathrm{m}^{3}\right]$ & Sample ratio \\
Spray zone 1 & $1.964 \times 10^{-5}$ & $1 / 500$ & $4.418 \times 10^{-5}$ & $1 / 500$ \\
Impeller zone 2 & $1.178 \times 10^{-4}$ & $1 / 500$ & $3.571 \times 10^{-4}$ & $1 / 500$ \\
Bulk zone 3 & $2.356 \times 10^{-4}$ & $1 / 500$ & $7.142 \times 10^{-4}$ & $1 / 500$ \\
Bulk zone 4 & $1.523 \times 10^{-3}$ & $1 / 500$ & $2.929 \times 10^{-3}$ & $1 / 500$ \\
\hline
\end{tabular}


In case I, due to the low ratio between liquid binder and dry powder, the rewetting rate is very low. Such a small liquid amount $(0.05 \mathrm{~g} / \mathrm{g}$ dry powder $)$ almost depletes the possibility of coalescence events, which significantly depends on the availability of liquid on the granule surface. Furthermore, owing to the bumping flow regime and relatively slow rotation speed, the breakage is ignored in case I. Therefore, the granulation process is simulated accounting for only two mechanisms: nucleation and layering.

Two kinetic parameters $\Phi_{c p}$ and $D_{e f f}$ were adjusted manually to match the experimental data. The parameters were selected as follows (Table 7). For a spherical geometry granule, the growth rate in Eq. 16 is insensitive to the critical packing fraction $\Phi_{c p}$, as discussed in Hounslow et al ${ }^{42}$.

Table 7: Parameters and prediction in case I

\begin{tabular}{ccccc}
\hline $\begin{array}{c}\text { Simulation } \\
\text { No. }\end{array}$ & $D_{\text {eff }}\left[\mathrm{m}^{2} \mathrm{~s}^{-1}\right]$ & $\Phi_{C P}[-]$ & $\begin{array}{c}d_{43}[\mu \mathrm{m}] \\
(450 \mathrm{~s})\end{array}$ & $H^{2}$ \\
\hline 1 & $5 \times 10^{-11}$ & 0.03 & 553.4 & 0.114 \\
2 & $2 \times 10^{-11}$ & 0.03 & 536.6 & 0.099 \\
3 & $2 \times 10^{-11}$ & 0.02 & 517.4 & 0.074 \\
4 & $9 \times 10^{-12}$ & 0.02 & 511.9 & 0.069 \\
5 & $9 \times 10^{-12}$ & 0.01 & 493.7 & 0.052 \\
6 & $7 \times 10^{-12}$ & 0.01 & 492.3 & 0.050 \\
7 & $7 \times 10^{-12}$ & 0.005 & 450.0 & 0.048 \\
8 & $7 \times 10^{-12}$ & 0.0025 & 450.0 & 0.050 \\
Measured & - & - & 385.7 & - \\
\hline
\end{tabular}

Parameter sensitivity was performed to investigate the uncertainty of the PBM prediction of granule size distribution (GSD) and binder size distribution (BSD) dependent on uncertainty in the model input (e.g. parameters in expression of granulation rate). The adjustment of parameters of the layering rate was found to produce a suitable fit of the GSD (Table 7).

Squared Hellinger Distance (SHD) $H^{2}{ }^{40}$ was used to quantify the goodness of fit (Table 7), which is defined as

$$
H^{2}(f, g)=\frac{1}{2} \int(\sqrt{f(x)}-\sqrt{g(x)})^{2} d x
$$

where $f(x), g(x)$ are probability density functions (PDF), describing the frequency of occurrence at size $x ; x$ is particle size.

Each granule starts to grow from a droplet as a nucleus or may be rewetted when it goes through the spray zone. Due to the low ratio between liquid binder and dry powder, only one 
mechanism, nucleation, is represented to transfer the mass of binder droplet into the second internal coordinate of granule, the mass of liquid. The nucleation event is able to increase the number of granules. In Fig. 9a, the number concentrations of granule in four compartments grow as a linear trend during first $150 \mathrm{~s}$, which is the addition time of liquid binder. The mass ratio $\eta$ of liquid binder mass to powder bed mass is defined as

$$
\eta_{L}=\frac{M_{L, t}}{M_{B, t}}
$$

$\mathrm{M}_{\mathrm{P}, \mathrm{t}}$ is total mass of liquid binder within powder bed at time $t$. In Fig. 9b, the agreement between the expected and simulated mass ratio $\eta_{L}$ shows that mass balance based on multicompartmental PBM is reliable. In Fig. 9c, the prediction of overall liquid content within granules over time shows that the liquid mass fraction in the granule population decreases from 1 (droplet nucleus) to approximately 0.0476 (input liquid/ (powder+ liquid)). The size of a granule in this study is calculated as the diameter of a granule at particle scale or average based granule size (i.e. $d_{43}$ ) at the granulator scale. In the multi-compartmental PBM, it is assumed no air is present, so the total volume of a granule is the sum of liquid component volume and solid component volume. The granule diameter is able to be estimated based on the assumption of spherical particle shape. The definition of $d_{43}$ is

$$
d_{43}=\frac{\int_{0}^{\infty} l^{4} n(l) d l}{\int_{0}^{\infty} l^{3} n(l) d l}
$$

In Fig 9d, the prediction of $d_{43}$ against time shows that the growth of granule size is not simple linear growth. The simulated results shown in Fig 9 use parameter set in test 7 in Table 7.

The droplet size and $\Phi_{C P}$ determine the maximal size the granule could achieve when it is saturated. $D_{\text {eff }}$ controls the layering rate of existing granules. In Fig 10, the comparison between simulated and measured ${ }^{36}$ granule size distribution (GSD) shows that multicompartmental PBM can provide a reasonable prediction for GSD. The increase of $\Phi_{C P}$ leads to an increase granule size (Table 7 and Fig. 10). GSD is solid mass weighted size distribution.

The mechanism of layering represents the transfer of the mass of the primary particles into the first internal coordinate of granule, the mass of solid. A mass ratio $\eta_{P}$ of primary particle mass to solid mass within powder bed can be defined as 


$$
\eta_{P}=\frac{M_{P, t}}{M_{B, t}}
$$

$\mathrm{M}_{\mathrm{P}, \mathrm{t}}$ is total mass of primary particles (dry powder) within powder bed at time $t ; M_{B, t}$ is total mass of solid (both primary particles and granules) within powder bed at time $t$. The mass ratio $\eta_{P}$ change over time is shown in Fig. 11, indicating that the population of powder bed transforms from dry powder a $\mathrm{t}=0 \mathrm{~s}$, to mixture of dry powder and wet granules at an intermediate stage and finally to wet granules after the depletion of the primary particle at $t$ $=420 \mathrm{~s}$. The increase of $D_{e f f}$ leads to an increased layering rate.

In Fig. 12, the mean liquid content and its standard deviation at different granule diameter shows that relatively small granules are saturated with powder and large granules are not saturated, exhibiting a high liquid content. The simulated liquid content distribution at different granule diameter shows that two different granules with the same size may have different liquid content, $\Phi_{C P}$ determines the minimum liquid content at different granule size in saturated status.

\section{Case II}

\section{Hydrodynamics and transport rate}

Due to low solid loading and high shear rate in case II, the solids are less heterogeneously distributed compared with case I throughout the granulator. The flow regime of roping flow where the bed rotates much faster and mixes vertically is seen in Fig.13 and Fig. 14. The solid concentration (Fig.14) exhibits the flow pattern features of roping regime, which tends to push the solid towards the outside of the bowl.

The compartment structure of case II is similar to case I shown in Fig. 6. The location of compartmental boundaries in case II is shown in Table 4. The temporal history of the volume flow rate and volume-based average solid volume fraction at each compartment are shown in Fig 15a and Fig. 15b, respectively. The variation range is less than 10\%. Therefore, we used averaged volume flow rates and averaged volume fractions for the solid phase to represent the transport rate among different compartments (Table 5).

Case II was simulated accounting for five different mechanisms: nucleation, rewetting, layering, coalescence and breakage. Four kinetic parameters were adjusted manually to match the experimental data. The values obtained are shown in Table 8. The critical packing 
fraction $\Phi_{c p}=0.3$ and $D_{e f f}=5 \times 10^{-10}$ were used to control the layering rate. Compartment volumes and sample sizes are shown in Table 6. The transport rates are shown in Table 5. For this case (case II: 4L DIOSNA granulator), parameter sensitivity (Table 8) shows that the adjustment of parameters in combination of layering, coalescence and breakage rate can provide a reasonable prediction of the GSD.

Table 8: Parameters and prediction in case II

\begin{tabular}{ccccccc}
\hline $\begin{array}{c}\text { Simulation } \\
\text { No. }\end{array}$ & $\begin{array}{c}D_{\text {eff }} \\
{\left[\mathrm{m}^{2} \mathrm{~s}^{-1}\right]}\end{array}$ & $\beta_{0}\left[\mathrm{~m}^{2.5} \mathrm{~s}^{-1}\right]$ & $S_{0}\left[\mathrm{~s}^{-1}\right]$ & $\begin{array}{c}d_{43}[\mu \mathrm{m}] \\
(240 \mathrm{~s})\end{array}$ & $\begin{array}{c}d_{43}[\mu \mathrm{m}] \\
(360 \mathrm{~s})\end{array}$ & $\begin{array}{c}d_{43}[\mu \mathrm{m}] \\
(480 \mathrm{~s})\end{array}$ \\
\hline 1 & $5 \times 10^{-10}$ & - & - & 457.1 & 462.3 & 462.1 \\
2 & $5 \times 10^{-10}$ & $5 \times 10^{-10}$ & - & 757.7 & 887.7 & 979.6 \\
3 & $5 \times 10^{-10}$ & $6 \times 10^{-10}$ & 0.03 & 775.6 & 902.6 & 1017.0 \\
4 & $5 \times 10^{-10}$ & $4 \times 10^{-10}$ & 0.03 & 649.3 & 745.3 & 832.7 \\
5 & $5 \times 10^{-10}$ & $4 \times 10^{-10}$ & - & 651.5 & 747.3 & 828.0 \\
6 & $5 \times 10^{-10}$ & $2 \times 10^{-10}$ & - & 558.8 & 624.8 & 679.5 \\
7 & $5 \times 10^{-10}$ & $4 \times 10^{-10}$ & 0.06 & 653.8 & 758.5 & 845.7 \\
8 & $5 \times 10^{-10}$ & $2 \times 10^{-10}$ & 0.06 & 557.1 & 626.3 & 689.6 \\
Measured & - & - & - & 441.1 & 439.4 & 823.0 \\
\hline
\end{tabular}

The mechanisms of nucleation and rewetting represent the transfer of the mass of the binder liquid into the second internal coordinate of the granule, the mass of liquid. The nucleation event is able to increase the number of granules and the rewetting is not able to change that number. The ratio of rewetting rate to nucleation rate is related to the surface area ratio of existing granules to primary particles. In Fig. 16a, the number concentration of granule in four compartments grow not as a linear trend owing to the coexistence of nucleation and rewetting. As the probability of a granule to be rewetted in the spray zone increases, nucleation rate decreases, and meanwhile, coalescence and breakage rates may change to adjust the total number of granules. After the liquid binder addition period of $240 \mathrm{~s}$, the number concentration is controlled under the combined action of coalescence and breakage events. The mechanism of layering represents the transfer of the mass of the primary particles into the first internal coordinate of granule, the mass of solid. In Fig. 16b, the prediction of mass ratio $\eta_{P}$ (Eq.21) over time shows that the population of the powder bed transforms from dry powder at the start, to a mixture of dry powder and wet granules at an intermediate stage and finally to wet granules after the depletion of the primary particle. The completion of layering (i.e. depletion of primary powder) with subsequent liquid droplets rewetting the existing granules. In Fig. 16c, the agreement between the expected and simulated mass ratio $\eta_{L}$ (Eq.19) shows that mass balance based on multi-compartmental PBM is reliable for case 
II. In Fig. 16d, the prediction of overall liquid content within granules over time shows that the liquid mass fraction in the granule population decreases from 1 (droplet nucleus) to approximately 0.2958 (input liquid/ (powder+liquid)).

In Fig. 17a, the simulated liquid content (liquid mass fraction) distributions at different granule diameter show that two different granules with the same size may have different internal coordinates. $\Phi_{C P}$ determines the minimum liquid content for different size granules in powder saturated states. In Fig 17b, the mean liquid mass fraction and standard deviation at different granule diameter shows that relatively small granules are saturated with powder and large granules have a high mean liquid content with a broader distribution. The cumulative GSD and cumulative BSD are shown in Fig. 17a and Fig. 17b prove that the parameters used in test 1 are fitted well at 240s.

In Fig 18, the predicted $d_{43}$ is compared to experimental data, which shows that the parameter set used in test 1 fitted well before 360s and the parameter set used in test 4, 5 and 7 fitted well at 480s. This is explained as follows. In the liquid addition stage (0 240s), a reliable prediction can be obtained using multi-compartmental PBM with nucleation, rewetting and layering mechanisms only. In the induction stage (240s $\sim 360 \mathrm{~s})$, the $d_{43}$ stays constant, which is consistent with the layering model reaching a saturated state. However in the latter stages (360-480s) coalescence and breakage mechanisms can provide a reasonable prediction of granule growth (Fig 19). To holistically include both effects a consolidation mechanism, which describes the expulsion of over-saturated liquid binder from pores inside the granules to the granule surface ${ }^{12}$, needs to be included in the model.

The validation and accuracy of numerical prediction from the compartmental PBM in this study depends on the validation and accuracy of the model set, including both CFD and PBM. Numerically, validation of our Monte Carlo code can be found in our previous publication ${ }^{40}$. Validation of the granulation mechanisms can be found in the granulation literature but specific validation remains more challenging due to the confounding contribution of multiple competing rate processes. This is where modelling can play a vital role to deconvolute the specific contributions of each mechanism, however validation will still be reliant on high resolution, multi-component granule characterisation. In terms of the CFD model, validation primarily focussed on prediction of the bulk flow regime. Although the transport flux from the current CFD model may have some deviation compared to real granulation conditions due to the limitation of Eulerian model of granular flow, the proposed model in this study 
provides a viable framework to progress compartmental modelling of high shear granulation and therefore incorporate changes due to equipment operating parameters and scales. In the future, the accuracy of compartmental PBM can be improved by enhanced Eulerian based model.

The influence of the flow flux between compartments on the PBM results is not considered in this study. However, it is of interest and essential to investigate the sensitivity of the solution and therefore how accurate the bulk flow model needs to be.

\section{Conclusions}

A compartmental PBM with spatial dependence has been developed for a HSG process. By analogy with multi-compartment crystallizers, a heuristic approach to compartmentalize a high shear granulator has been applied to two real granulation cases, 1.9L MirPo high shear granulator and $4 \mathrm{~L}$ DIOSNA high shear granulator based on CFD simulation. The compartmental structure is based on solid-based hydrodynamic information obtained from CFD simulations to include the overall flow pattern, granule velocity and solids concentration distribution. The size, location and volume of each compartment are estimated from CFD and qualitative analysis (spray zone and chopper zone) using the physical granulator dimensions. After realizing the compartmentalization of the high shear granulator into meso-scale CSTR's (continuous stirred-tank reactor) and identifying the boundary of meso-scale CSTRs, we estimate the transportation rate among meso-scale CSTRs to connect these CSTRs for subsequent development of a distributed macroscopic model (PBM).

The two lab-scale high shear granulation processes are modeled using a compartmental PBM. The advantage of compartmental PBM is different spatial dependent mechanisms can be included in the PBM to describe granule growth. The CVMC approach has been implemented to solve the multi-compartment PBEs, and it is capable of solving multi-dimensional PBEs with both internal and external coordinates which shows it can be easily extended to add more informative coordinates of granules in further development. Experimental data are used to validate the novel model. In is concluded that

1) For the low liquid content study case (case I: 1.9L MiPro granulator), parameter sensitivity shows that the adjustment of parameters in layering rate can provide a fitted prediction. Increasing $\Phi_{C P}$ leads to an increase granule size. Increasing $D_{e f f}$ leads to an increased layering rate. 
2) For the high liquid content study case (case II: 4L DIOSNA granulator), parameter sensitivity shows that the adjustment of parameters for layering, coalescence and breakage rate can provide a reasonable prediction, although a consolidation mechanism to allow transition between the layering dominated induction phase and the coalescence dominated rapid growth phase is needed.

The reasonable agreement indicates the potential of compartmental PBM for scale-up and the importance of understanding the dynamics of the granulation process. Further improvements of such a model are closely related to new developments in the physical understanding of granulation mechanisms, and improved capability in measured multi-dimensional variables experimentally for model calibration and validation.

\section{Acknowledgment}

The authors are grateful to AstraZeneca for funding

\section{Appendix}

The main model equations used to simulate the non-reacting isothermal solid-gas suspension in the high shear granulator are given ${ }^{59,60}$ :

Continuity equations:

$$
\begin{gathered}
\frac{\partial\left(\alpha_{g} \rho_{g}\right)}{\partial t}+\nabla\left(\alpha_{g} \rho_{g} \vec{u}_{g}\right)=0 \\
\frac{\partial\left(\alpha_{s} \rho_{s}\right)}{\partial t}+\nabla\left(\alpha_{s} \rho_{s} \vec{u}_{s}\right)=0 \\
\alpha_{s}+\alpha_{g}=1
\end{gathered}
$$

Momentum equations:

$$
\begin{aligned}
& \frac{\partial\left(\alpha_{g} \rho_{g} \vec{u}_{g}\right)}{\partial t}+\nabla\left(\alpha_{g} \rho_{g} \vec{u}_{g} \vec{u}_{g}\right)=-\alpha_{g} \nabla P+\nabla \overline{\bar{\tau}}_{g}-\beta\left(u_{g}-u_{s}\right)+\alpha_{g} \rho_{g} \vec{g} \\
& \frac{\partial\left(\alpha_{s} \rho_{s} \vec{u}_{s}\right)}{\partial t}+\nabla\left(\alpha_{s} \rho_{s} \vec{u}_{s} \vec{u}_{s}\right)=-\alpha_{s} \nabla P-\nabla P_{s}+\nabla \overline{\bar{\tau}}_{s}+\beta\left(u_{g}-u_{s}\right)+\alpha_{s} \rho_{s} \vec{g}
\end{aligned}
$$

where: 


$$
\begin{gathered}
\overline{\bar{\tau}}_{k}=\left(\lambda_{k}-\frac{2}{3} \mu_{k}\right)\left(\nabla \cdot \vec{u}_{k}\right) \overline{\bar{I}}+2 \mu_{k} \overline{\bar{S}}_{k} \\
\overline{\bar{S}}_{k}=\frac{1}{2}\left(\nabla \vec{u}_{k}+\left(\nabla \vec{u}_{k}\right)^{T}\right)
\end{gathered}
$$

$k$ represents solid or gas phase.

The granular temperature was obtained by solving the Pseudo kinetic energy equation given by follows ${ }^{61}$ :

$$
\frac{3}{2}\left[\frac{\partial\left(\alpha_{s} \rho_{s} \Theta_{S}\right)}{\partial t}+\nabla\left(\alpha_{s} \rho_{s} \Theta_{S}\right) \vec{u}_{s}\right]=\left(-P_{S} \overline{\bar{I}}+\overline{\bar{\tau}}_{s}\right): \nabla \vec{u}_{s}+\nabla\left(\kappa_{\Theta_{S}} \nabla \Theta_{S}\right)-\gamma_{\Theta_{S}}+\phi_{g s}
$$

The various closure and constitutive relations used in the model are given in Table A1.

Table A1: Constitutive relations for gas-solid flow

\section{Solids pressure}

$$
P_{s}=\alpha_{s} \rho_{s} \Theta_{s}+2 \rho_{s}\left(1+e_{s s}\right) \alpha_{s}^{2} g_{0, s s} \Theta_{s}
$$

Solids shear viscosity

$$
\mu_{S}=\mu_{S, \text { col }}+\mu_{S, k i n}+\mu_{S, f r}
$$

Collisional viscosity ${ }^{62}$

$$
\mu_{S, c o l}=\frac{4}{5} \alpha_{S} \rho_{S} d_{S} g_{0, S S}\left(e_{S S}+1\right)\left(\frac{\Theta_{S}}{\pi}\right)^{1 / 2}
$$

Kinetic viscosity ${ }^{63}$

$\mu_{S, k i n}=\frac{\alpha_{S} \rho_{S} d_{S} \sqrt{\theta_{S} \pi}}{6\left(3-e_{S S}\right)}\left[1+\frac{2}{5}\left(e_{S S}+1\right)\left(3 e_{S S}-1\right) \alpha_{S} g_{0, S S}\right]$

Friction viscosity ${ }^{64}$

$\mu_{S, f r}=\frac{P_{S} \sin \phi}{2 \sqrt{I_{2 D}}}$

Bulk viscosity ${ }^{65}$

$$
\lambda_{S}=\frac{4}{3} \alpha_{S} \rho_{S} d_{S} g_{0, S S}\left(e_{S S}+1\right)\left(\frac{\Theta_{S}}{\pi}\right)^{1 / 2}
$$

Radial distribution function

$g_{0, s s}=\left(1-\left(\frac{\alpha_{s}}{\alpha_{s, \max }}\right)^{1 / 3}\right)^{-1}$

Gas-solid drag coefficient ${ }^{66}$

$$
\begin{aligned}
& \beta=\frac{3}{4} C_{D} \frac{\left(1-\alpha_{S}\right) \alpha_{S} \rho_{g}\left|\vec{u}_{g}-\vec{u}_{s}\right|}{D_{P}}\left(1-\alpha_{S}\right)^{-2.65} \\
& v_{r, s}=0.5\left(A-0.06 R e_{S}+\sqrt{\left(0.06 R e_{S}\right)^{2}+0.12 R e_{S}(2 B-A)+A^{2}}\right) \\
& A=\alpha_{g}^{4.14}, \quad\left\{\begin{array}{c}
B=0.8 \alpha_{g}^{1.28}\left(\alpha_{g} \leq 0.85\right) \\
B=\alpha_{g}^{2.65}\left(\alpha_{g}>0.85\right)
\end{array}\right. \\
& C_{D}=\left(0.63+\frac{4.8}{\sqrt{R e_{S} / v_{r, s}}}\right)^{2}
\end{aligned}
$$




$$
R e_{s}=\frac{d_{s} \rho_{g}\left|\vec{u}_{g}-\vec{u}_{s}\right|}{\mu_{g}}
$$

Diffusion coefficient of granular energy ${ }^{67}$

Collisional energy dissipation ${ }^{67}$

$$
\begin{gathered}
\kappa_{\Theta_{S}}=\frac{150 \rho_{S} d_{S}\left(\pi \Theta_{S}\right)^{\frac{1}{2}}}{384\left(e_{S S}+1\right) g_{0, S S}}\left[1+\frac{6}{5} \alpha_{S} g_{0, S S}\left(e_{S S}+1\right)\right]^{2} \\
+2 \alpha_{S}{ }^{2} \rho_{S} d_{S} g_{0, S S}\left(e_{S S}+1\right)\left(\frac{\Theta_{S}}{\pi}\right)^{\frac{1}{2}}
\end{gathered}
$$

$$
\gamma_{\Theta_{S}}=\frac{12\left(1-e_{S S}{ }^{2}\right) g_{0, S S}}{d_{S} \pi^{1 / 2}} \alpha_{S}^{2} \rho_{S} \Theta_{S}^{3 / 2}
$$

\section{Boundary and simulation conditions}

For the gas phase, the velocity at the wall (vessel wall and the impeller surface) was assumed zero (no slip condition). In this work, a partial slip model is employed. It was initially presented by $\mathrm{Tu}$ and Fletcher ${ }^{68}$ and applied for modelling particle velocity at the wall in a high shear granulator using two fluid model by Darelius et al ${ }^{50}$. The partial slip model can cover the whole range between two extremes (no slip and free slip). The expression is:

$$
a \varphi_{w}-\left.b \frac{\partial \varphi}{\partial \eta}\right|_{w}=0
$$

$\varphi_{w}=\left[u_{p w}^{N}, u_{p w}^{T}\right]$ is a vector containing the normal and tangential solid velocities at the wall, respectively, $\eta$ is normal direction of the wall directed into flow regime, $a, b$ are positive constants with different values depending on if the normal or tangential direction is considered. The constants $a$ and $b$ are functions of the coefficient of wall restitutions in the normal and tangential directions, respectively. They can be calculated as

$$
\begin{aligned}
& a_{N}=A_{N}-B_{N}, b_{N}=A_{N} h \\
& a_{T}=A_{T}-B_{T}, b_{T}=A_{T} h
\end{aligned}
$$

Where: 


$$
\begin{array}{ll}
A_{N}=\left[\frac{1+e_{p}^{N}\left(-e_{p}^{N}\right)}{2}\right]^{1 / 2}, & A_{T}=\left[\frac{1+\left(e_{p}^{T}\right)^{2}}{2}\right]^{\frac{1}{2}} \\
B_{N} & =\left[\frac{e_{p}^{N}\left(1-e_{p}^{N}\right)}{1+e_{p}^{N}}\right]^{1 / 2}, \quad B_{T}=\left[\frac{e_{p}^{N}+\left(e_{p}^{T}\right)^{2}}{1+e_{p}^{N}}\right]^{1 / 2}
\end{array}
$$

$e_{p}^{N}, e_{p}^{T}$ represent the coefficients of wall restitution in the normal and tangential direction, $h$ is the thickness of the computational cell next to the boundary. The ratio $a / b$ is a measure of the degree of slip. In the tangential direction an infinite ratio corresponds to no slip and a zero ratio represents free slip. A partial slip boundary condition was used for the solid phase, $e_{p}^{N}=e_{p}^{T}=0.5$ in this study. The top lid of the vessel was not closed but connected to the surrounding atmosphere and hence, it was modelled using a constant pressure boundary condition.

\section{References}

1. Reynolds GK, Le PK, Nilpawar AM. Chapter 1 High shear granulation. In: A.D. Salman MJH, Seville JPK, eds. Handbook of Powder Technology. Vol Volume 11: Elsevier Science B.V.; 2007:3-19.

2. Faure A, York P, Rowe RC. Process control and scale-up of pharmaceutical wet granulation processes: a review. European Journal of Pharmaceutics and Biopharmaceutics. 11// 2001;52(3):269-277.

3. Benali M, Gerbaud V, Hemati M. Effect of operating conditions and physicochemical properties on the wet granulation kinetics in high shear mixer. Powder Technology. 3/5/ 2009;190(1-2):160-169.

4. Stahl H. Comparing different granulation Techniques. Pharmaceutical Technology Europe. 2004;11:23-33.

5. Snow RH AT, Ennis BJ, Litster JD. Size reduction and size enlargement. In: Perry RH GDE, ed. Perry's Chemical Engineers' Handbook. USA McGraw-Hill; 1997.

6. Mangwandi C, Adams MJ, Hounslow MJ, Salman AD. Influence of fill factor variation in high shear granulation on the post granulation processes: Compression and tablet properties. Powder Technology. 9// 2014;263:135-141.

7. Chan EL, Reynolds GK, Gururajan B, Hounslow MJ, Salman AD. Blade-granule bed stress in a cylindrical high shear granulator: I-Online measurement and characterisation. Chemical Engineering Science. 2/4/ 2013;86:38-49.

8. Reynolds GK, Nilpawar AM, Salman AD, Hounslow MJ. Direct measurement of surface granular temperature in a high shear granulator. Powder Technology. 2/22/ 2008;182(2):211-217.

9. Washino K, Tan HS, Hounslow MJ, Salman AD. A new capillary force model implemented in micro-scale CFD-DEM coupling for wet granulation. Chemical Engineering Science. 4/19/2013;93:197-205. 
10. Khalilitehrani M, Abrahamsson PJ, Rasmuson A. Modeling dilute and dense granular flows in a high shear granulator. Powder Technology. 9// 2014;263:45-49.

11. Nguyen D, Rasmuson A, Niklasson Björn I, Thalberg K. CFD simulation of transient particle mixing in a high shear mixer. Powder Technology. 5// 2014;258:324-330.

12. Iveson SM, Litster JD, Hapgood K, Ennis BJ. Nucleation, growth and breakage phenomena in agitated wet granulation processes: a review. Powder Technology. 6/4/ 2001;117(1-2):3-39.

13. Sanders CFW, Willemse AW, Salman AD, Hounslow MJ. Development of a predictive high-shear granulation model. Powder Technology. 11/30/ 2003;138(1):1824.

14. Darelius A, Rasmuson A, Björn IN, Folestad S. High shear wet granulation modelling - a mechanistic approach using population balances. Powder Technology. 12/13/ 2005;160(3):209-218.

15. Biggs CA, Sanders C, Scott AC, et al. Coupling granule properties and granulation rates in high-shear granulation. Powder Technology. 2/19/ 2003;130(1-3):162-168.

16. Le PK, Avontuur P, Hounslow MJ, Salman AD. The kinetics of the granulation process: Right from the early stages. Powder Technology. 1/31/2009;189(2):149-157.

17. Oullion M, Reynolds GK, Hounslow MJ. Simulating the early stage of high-shear granulation using a two-dimensional Monte-Carlo approach. Chemical Engineering Science. 2// 2009;64(4):673-685.

18. Darelius A, Brage H, Rasmuson A, Niklasson Björn I, Folestad S. A volume-based multi-dimensional population balance approach for modelling high shear granulation. Chemical Engineering Science. 4// 2006;61(8):2482-2493.

19. Ramachandran R, Immanuel CD, Stepanek F, Litster JD, Doyle Iii FJ. A mechanistic model for breakage in population balances of granulation: Theoretical kernel development and experimental validation. Chemical Engineering Research and Design. 4// 2009;87(4):598-614.

20. Braumann A, Goodson MJ, Kraft M, Mort PR. Modelling and validation of granulation with heterogeneous binder dispersion and chemical reaction. Chemical Engineering Science. 9// 2007;62(17):4717-4728.

21. Braumann A, Kraft $M$. Incorporating experimental uncertainties into multivariate granulation modelling. Chemical Engineering Science. 2/1/ 2010;65(3):1088-1100.

22. Braumann A, Kraft M, Mort PR. Parameter estimation in a multidimensional granulation model. Powder Technology. 1/25/ 2010;197(3):196-210.

23. Lister JD, Smit DJ, Hounslow MJ. Adjustable discretized population balance for growth and aggregation. AIChE Journal. 1995;41(3):591-603.

24. Verkoeijen D, A. Pouw G, M. H. Meesters G, Scarlett B. Population balances for particulate processes-a volume approach. Chemical Engineering Science. 6// 2002;57(12):2287-2303.

25. Michaels JN. Toward rational design of powder processes. Powder Technology. 11/30/ 2003;138(1):1-6.

26. Kramer HJM, Dijkstra JW, Neumann AM, ÓMeadhra R, van Rosmalen GM. Modelling of industrial crystallizers, a compartmental approach using a dynamic flow-sheeting tool. Journal of Crystal Growth. 9/1/ 1996;166(1-4):1084-1088.

27. Bermingham SK, Kramer HJM, van Rosmalen GM. Towards on-scale crystalliser design using compartmental models. Computers \& Chemical Engineering. 3/15/ 1998;22, Supplement 1:S355-S362.

28. Kramer HJM, Bermingham SK, van Rosmalen GM. Design of industrial crystallisers for a given product quality. Journal of Crystal Growth. 3// 1999;198-199, Part 1:729737. 
29. Kramer HJM, Dijkstra JW, Verheijen PJT, Van Rosmalen GM. Modeling of industrial crystallizers for control and design purposes. Powder Technology. 3/20/ 2000;108(2-3):185-191.

30. Zauner R, Jones AG. On the influence of mixing on crystal precipitation processesapplication of the segregated feed model. Chemical Engineering Science. 3// 2002;57(5):821-831.

31. Rigopoulos S, Jones A. A hybrid CFD-reaction engineering framework for multiphase reactor modelling: basic concept and application to bubble column reactors. Chemical Engineering Science. 7// 2003;58(14):3077-3089.

32. Kougoulos E, Jones AG, Wood-Kaczmar M. CFD Modelling of Mixing and Heat Transfer in Batch Cooling Crystallizers: Aiding the Development of a Hybrid Predictive Compartmental Model. Chemical Engineering Research and Design. 1// 2005;83(1):30-39.

33. Bezzo F, Macchietto S. A general methodology for hybrid multizonal/CFD models: Part II. Automatic zoning. Computers \& Chemical Engineering. 4/15/ 2004;28(4):513-525.

34. Yu X. An in-silico model of granulation. PhD Thesis, Sheffield University. 2012.

35. Yu X, Hounslow MJ, Reynolds GK. Representing spray zone with cross flow as a well-mixed compartment in a high shear granulator. Powder Technology. 9// 2016;297:429-437.

36. Niklasson Björn I, Jansson A, Karlsson M, Folestad S, Rasmuson A. Empirical to mechanistic modelling in high shear granulation. Chemical Engineering Science. 7// 2005;60(14):3795-3803.

37. Litster JD, Hapgood KP, Michaels JN, Sims A, Roberts M, Kameneni SK. Scale-up of mixer granulators for effective liquid distribution. Powder Technology. 4/29/ 2002;124(3):272-280.

38. Liffman K. A direct simulation Monte-Carlo method for cluster coagulation. Journal of Computational Physics. 1992/05/01 1992;100(1):116-127.

39. Maisels A, Einar Kruis F, Fissan H. Direct simulation Monte Carlo for simultaneous nucleation, coagulation, and surface growth in dispersed systems. Chemical Engineering Science. 6// 2004;59(11):2231-2239.

40. Yu X, Hounslow MJ, Reynolds GK. Accuracy and optimal sampling in Monte Carlo solution of population balance equations. AIChE Journal. 2015;61(8):2394-2402.

41. Hounslow MJ, Pearson JMK, Instone T. Tracer studies of high-shear granulation: II. Population balance modeling. AIChE Journal. 2001;47(9):1984-1999.

42. Hounslow MJ, Oullion M, Reynolds GK. Kinetic models for granule nucleation by the immersion mechanism. Powder Technology. 1/31/ 2009;189(2):177-189.

43. Chan EL, Washino K, Ahmadian H, et al. Dem investigation of horizontal high shear mixer flow behaviour and implications for scale-up. Powder Technology. 1// 2015;270, Part B:561-568.

44. Hassanpour A, Pasha M, Susana L, Rahmanian N, Santomaso AC, Ghadiri M. Analysis of seeded granulation in high shear granulators by discrete element method. Powder Technology. 4// 2013;238:50-55.

45. Nakamura H, Fujii H, Watano S. Scale-up of high shear mixer-granulator based on discrete element analysis. Powder Technology. 2// 2013;236:149-156.

46. Remy B, Canty TM, Khinast JG, Glasser BJ. Experiments and simulations of cohesionless particles with varying roughness in a bladed mixer. Chemical Engineering Science. 8/15/ 2010;65(16):4557-4571. 
47. Nakamura H, Miyazaki Y, Sato Y, Iwasaki T, Watano S. Numerical analysis of similarities of particle behavior in high shear mixer granulators with different vessel sizes. Advanced Powder Technology. 9// 2009;20(5):493-501.

48. Hassanpour A, Kwan CC, Ng BH, et al. Effect of granulation scale-up on the strength of granules. Powder Technology. 1/31/2009;189(2):304-312.

49. $\mathrm{Ng} \mathrm{BH}$, Ding YL, Ghadiri M. Modelling of dense and complex granular flow in high shear mixer granulator-A CFD approach. Chemical Engineering Science. 8/15/ 2009;64(16):3622-3632.

50. Darelius A, Rasmuson A, van Wachem B, Niklasson Björn I, Folestad S. CFD simulation of the high shear mixing process using kinetic theory of granular flow and frictional stress models. Chemical Engineering Science. 4// 2008;63(8):2188-2197.

51. Abrahamsson PJ, Björn IN, Rasmuson A. Parameter study of a kinetic-frictional continuum model of a disk impeller high-shear granulator. Powder Technology. 4// 2013;238:20-26.

52. Darelius A, Remmelgas J, Rasmuson A, van Wachem B, Björn IN. Fluid dynamics simulation of the high shear mixing process. Chemical Engineering Journal. 11/1/ 2010;164(2-3):418-424.

53. Vasquez S, Ivanov V. A phase coupled method for solving multiphase problems on unstructured meshes. Paper presented at: Proceedings of ASME FEDSM'00: ASME 2000 fluids engineering division summer meeting, Boston2000.

54. Courant R, Friedrichs K, Lewy H. On the partial difference equations of mathematical physics. IBM J. Res. Dev. 1967;11(2):215-234.

55. Yu X, Makkawi Y, Ocone R, Huard M, Briens C, Berruti F. A CFD study of biomass pyrolysis in a downer reactor equipped with a novel gas-solid separator - I: Hydrodynamic performance. Fuel Processing Technology. 10// 2014;126:366-382.

56. $\mathrm{Yu} \mathrm{X}$, Hassan $\mathrm{M}$, Ocone R, Makkawi Y. A CFD study of biomass pyrolysis in a downer reactor equipped with a novel gas-solid separator-II thermochemical performance and products. Fuel Processing Technology. 5// 2015;133:51-63.

57. Zhou YC, Yu AB, Bridgwater J. Segregation of binary mixture of particles in a bladed mixer. Journal of Chemical Technology \& Biotechnology. 2003;78(2-3):187193.

58. Zhou YC, Yu AB, Stewart RL, Bridgwater J. Microdynamic analysis of the particle flow in a cylindrical bladed mixer. Chemical Engineering Science. 3// 2004;59(6):1343-1364.

59. Anderson TB, Jackson R. Fluid Mechanical Description of Fluidized Beds. Equations of Motion. Industrial \& Engineering Chemistry Fundamentals. 1967/11/01 1967;6(4):527-539.

60. Mellin P, Yu X, Yang W, Blasiak W. Influence of Reaction Atmosphere (H2O, N2, $\mathrm{H} 2, \mathrm{CO} 2, \mathrm{CO})$ on Fluidized-Bed Fast Pyrolysis of Biomass Using Detailed Tar Vapor Chemistry in Computational Fluid Dynamics. Industrial \& Engineering Chemistry Research. 2015/08/26 2015;54(33):8344-8355.

61. Ding J, Gidaspow D. A bubbling fluidization model using kinetic theory of granular flow. AIChE Journal. 1990;36(4):523-538.

62. Gidaspow D, Bezburuah R, Ding J. Hydrodynamics of circulating fluidized beds: Kinetic theory approach 1991.

63. Syamlal M, Rogers W. MFIX documentation: Theory guide. 1993.

64. Schaeffer DG. Instability in the evolution equations describing incompressible granular flow. J Differ Equations. 1987/01/01 1987;66(1):19-50. 
65. Lun CKK, Savage SB, Jeffrey DJ, Chepurniy N. Kinetic theories for granular flow: inelastic particles in Couette flow and slightly inelastic particles in a general flowfield. J Fluid Mech. 1984;140:223-256.

66. Gera D, Gautam M, Tsuji Y, Kawaguchi T, Tanaka T. Computer simulation of bubbles in large-particle fluidized beds. Powder Technology. 7/15/ 1998;98(1):38-47.

67. Lun CKK, Savage SB, Jeffrey DJ, Chepurniy N. Kinetic Theories for Granular Flow Inelastic Particles in Couette-Flow and Slightly Inelastic Particles in a General Flowfield. J Fluid Mech. 1984;140(Mar):223-256.

68. Tu JY, Fletcher CAJ. Numerical computation of turbulent gas-solid particle flow in a $90^{\circ}$ bend. AIChE Journal. 1995;41(10):2187-2197.

\section{List of figures}

Fig 1: The computational domain of (a) 1.9L MiPro granulator (b) 4 L DIOSNA granulator

Fig 2: MMC algorithm

Fig 3: Droplet size distribution

Fig 4: Velocity vector for solid phase in case I (a) top view $(z=H / 11)(b)$ cross-section $(x=0)$ at steady condition, the velocity of the blade tip is approximate $7 \mathrm{~m} / \mathrm{s}$.

Fig 5: Contour plot of solid concentration at cross-section (a) $x=0$, and top view of (b) $z=H / 11(c) z=3 H / 11$ (d) $z=5 H / 11$ (e) $z=7 H / 11$ (f) $z=9 H / 11$ (g) $z=11 H / 11$ at steady condition

Fig 6: Compartment structure of (a) the location of compartments (b) top view of rotated compartment 2 and 3 (c) the connectivity diagram

Fig 7: The impact of sample number on overall net transport rate in case II

Fig 8: Temporal history of (a) volume flow rate for solid phase from compartment $i$ to $j$ in case I, 2 represents Impeller zone 2; 3 represents Bulk zone 3; 4 represents Bulk zone 4. (b) solid volume fraction in Impeller zone 2, Bulk zone 3, rotor part of Bulk zone 4 and stator part of Bulk zone 4 in case I

Fig 9: Temporal evolution of (a) predicted number concentration of granules for four compartments $(b)$ the expected $(-)$ and simulated $(\bullet)$ mass ratio $\eta_{L}(c)$ predicted overall liquid content within granules (d) predicted of granule mean size $d_{43}$ using test 7 in case $I$

Fig 10: Comparison between the simulated $(-)$ and measured $(\bullet)$ granule size distribution (GSD) at 450 s at $\Phi_{C P}=0.005-0.03$ in case I

Fig 11: Predicted mass ratio $\eta_{P}$ against time at 450s using test 2 and 1 in case 1

Fig 12: Predicted mean liquid content and its standard deviation against granule diameter at 450s using (a) $D_{\text {eff }}=2 \times 10^{-11}$, test 2 (b) $D_{\text {eff }}=5 \times 10^{-11}$, test 1 ; the error bar indicates the standard deviation of liquid mass fraction at each granule diameter; the target liquid mass fraction in these graphs with a dashed line in case I 
Fig 13: Velocity vector for solid phase (a) top view $(z=10 \mathrm{H} / 113)$ (b) cross-section $(x=0)$ at steady condition in case II, the velocity of the blade tip is approximate $14 \mathrm{~m} / \mathrm{s}$.

Fig 14: Countor plot of solid concentration profile (a) cross-section of $x=0$, and top view of $(b)$ $z=10 \mathrm{H} / 113$ (c) $z=30 \mathrm{H} / 113$ (d) $z=50 \mathrm{H} / 113$ (e) $z=70 \mathrm{H} / 113$ (f) $z=90 \mathrm{H} / 113$ (g) $z=110 \mathrm{H} / 113$ at steady condition in case II

Fig 15: Temporal history of (a) volume flow rate for solid phase from compartment $i$ to $j$ in case II, (2 represents Impeller zone 2; 3 represents Bulk zone 3; 4 represents Bulk zone 4). (b) solid volume fraction in Impeller zone 2, Bulk zone 3 and Bulk zone 4 for case II

Fig 16: Temporal evolution of (a) predicted number concentration of granules for four compartments (b) predicted mass ratio $\eta_{P}(c)$ the expected $(-)$ and simulated $(\bullet)$ mass ratio $\eta_{L}(d)$ predicted overall liquid content within granules using test 1 in case II.

Fig 17: (a) predicted liquid content (liquid mass fraction) distribution against granule size (contour plot) [The weight of the contour shading indicates relative number density.] (b) predicted mean liquid mass fraction and standard deviation against granule diameter [The error bar indicates the standard deviation of liquid mass fraction at each granule diameter] (c) comparison the simulated (-) and measured (•) cumulative GSD (d) comparison the simulated $(-)$ and measured $(\bullet)$ cumulative BSD at 240s using test 1 in case II.

Fig 18: Comparison between the simulated $(-)$ and measured $(\bullet)$ granule mean size $d_{43}$ against time in case II.

Fig 19: Comparison between the simulated $(-)$ and measured $(\bullet)$ cumulative probability distribution based on (a) GSD (b) BSD at 480s using test 4, 5 and 7 in case II. 

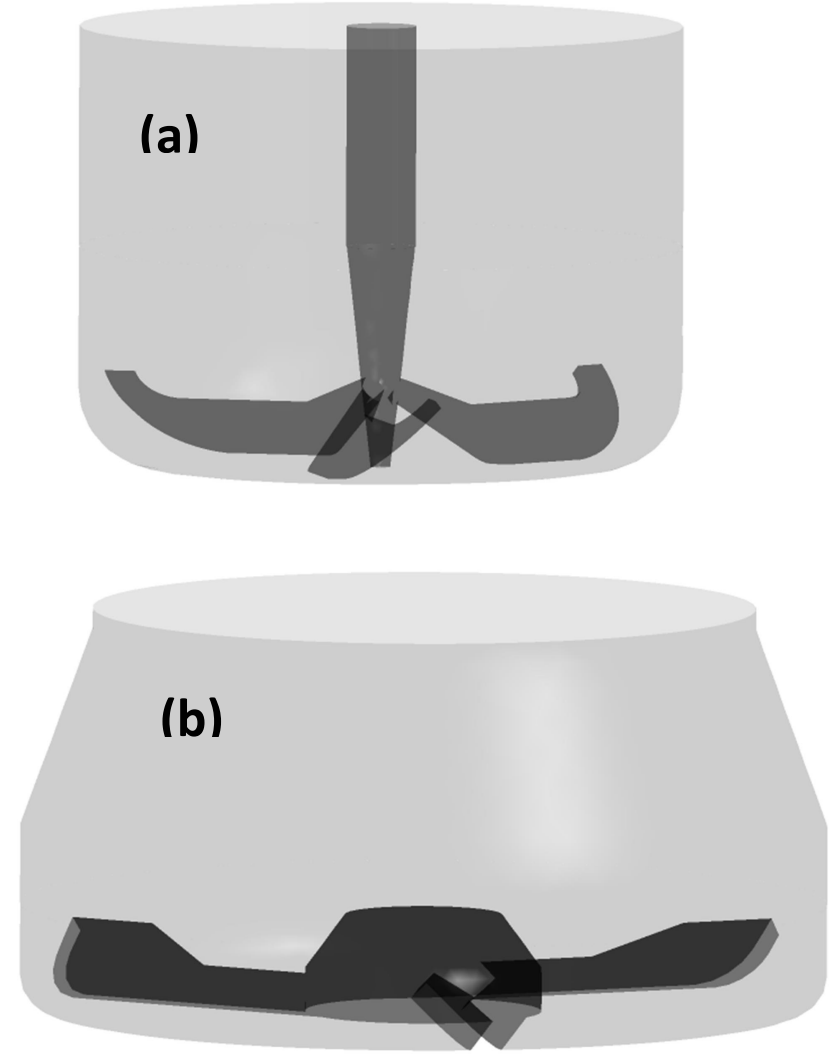

Fig. 1 The computational domain of (a) 1.9L MiPro granulator (b) 4L DIOSNA granulator 


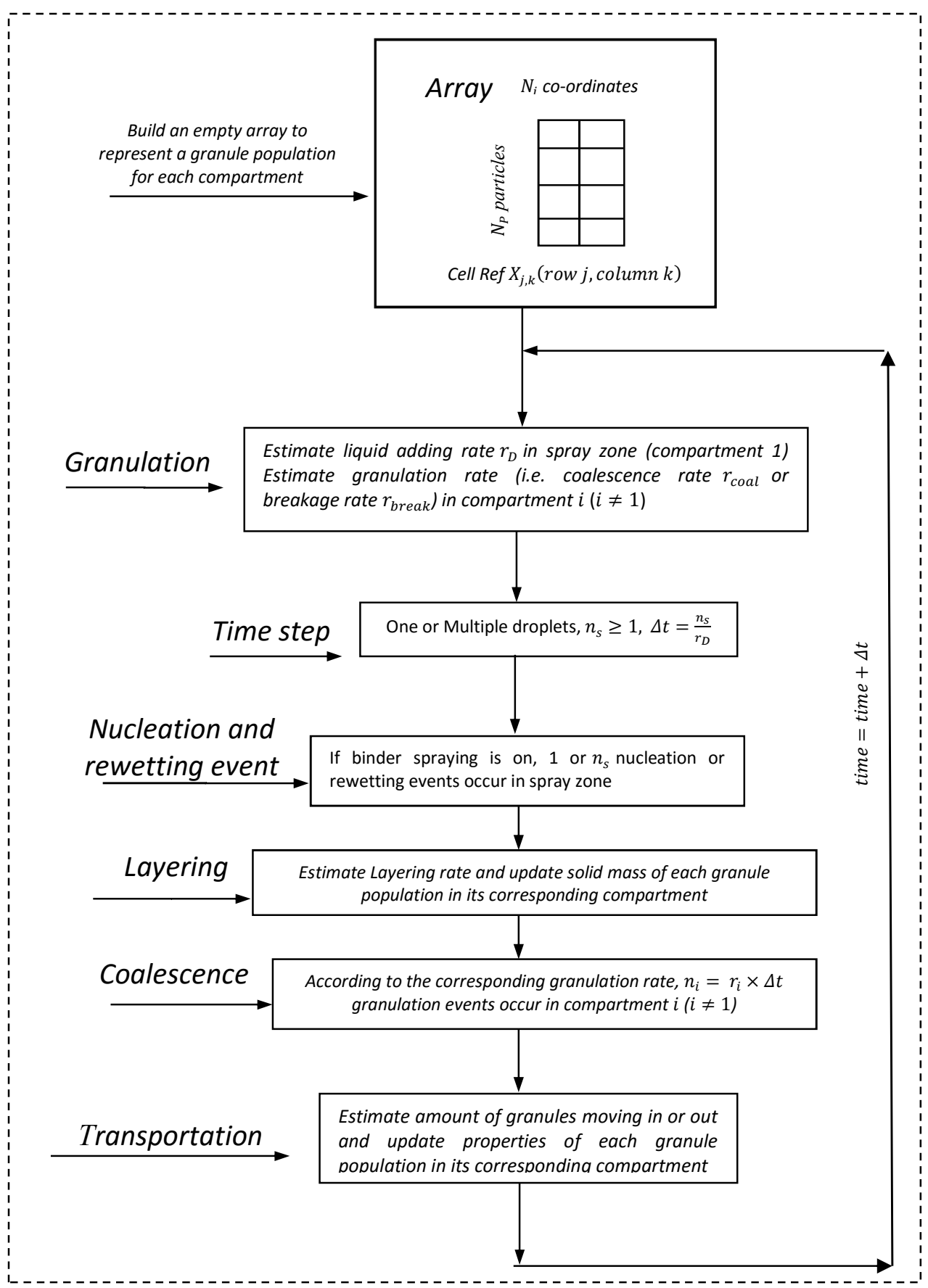

Fig2: MMC algorithm 


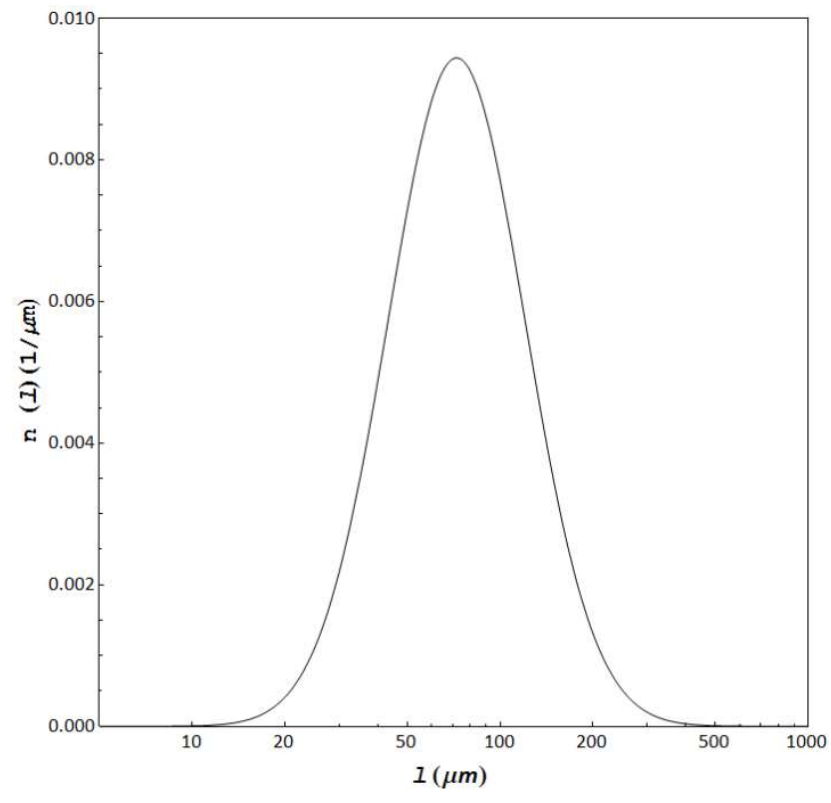

Fig3: Droplet size distribution
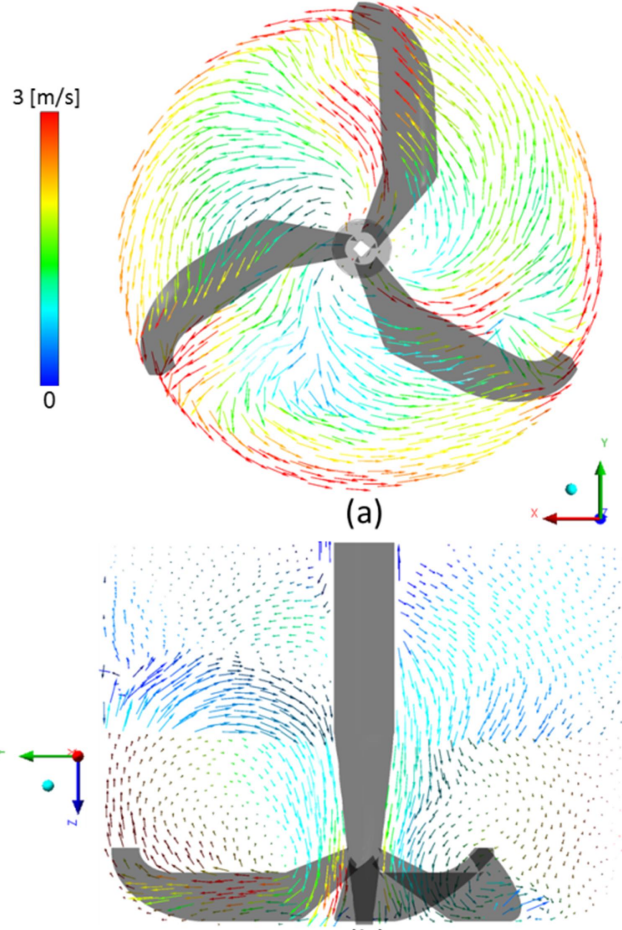

(b)

Fig 4 Velocity vector for solid phase in case I (a) top view $(z=H / 11)(b)$ cross-section $(x=0)$ at steady condition 


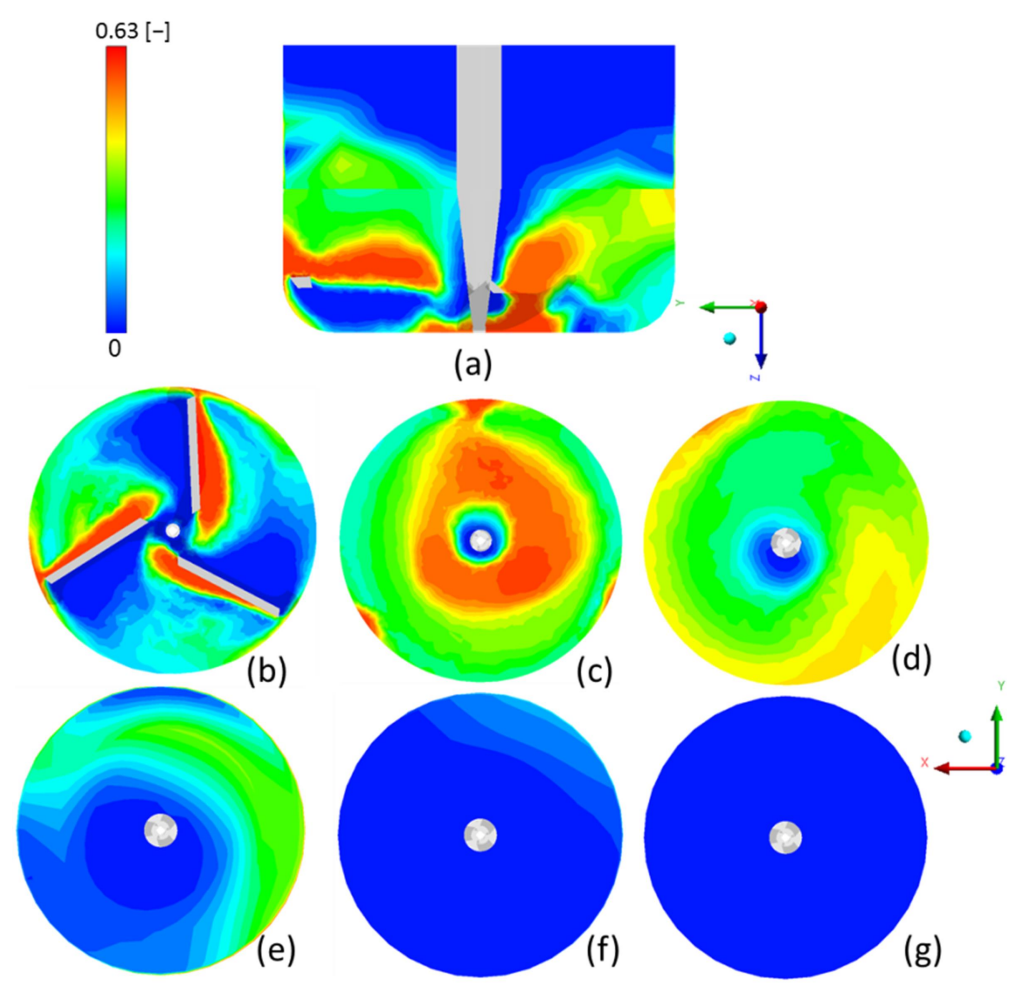

Fig 5 Contour plot of solid concentration at cross-section (a) $x=0$, and top view of (b) $z=H / 11(c) z=3 H / 11$ (d) $z=5 H / 11$ (e) $z=7 H / 11$ (f) $z=9 H / 11$ (g) $z=11 H / 11$ at steady condition 


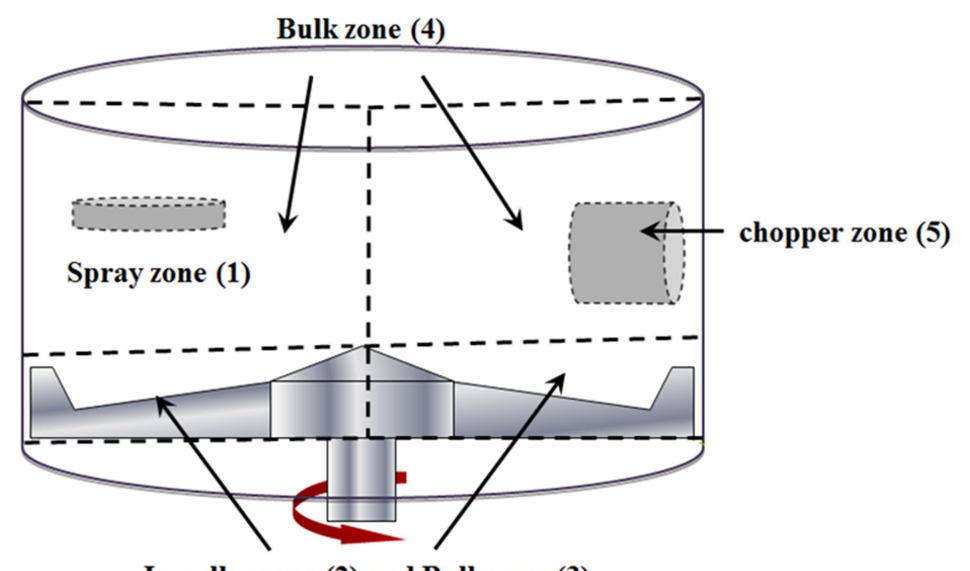

Impeller zone (2) and Bulk zone (3)

(a)
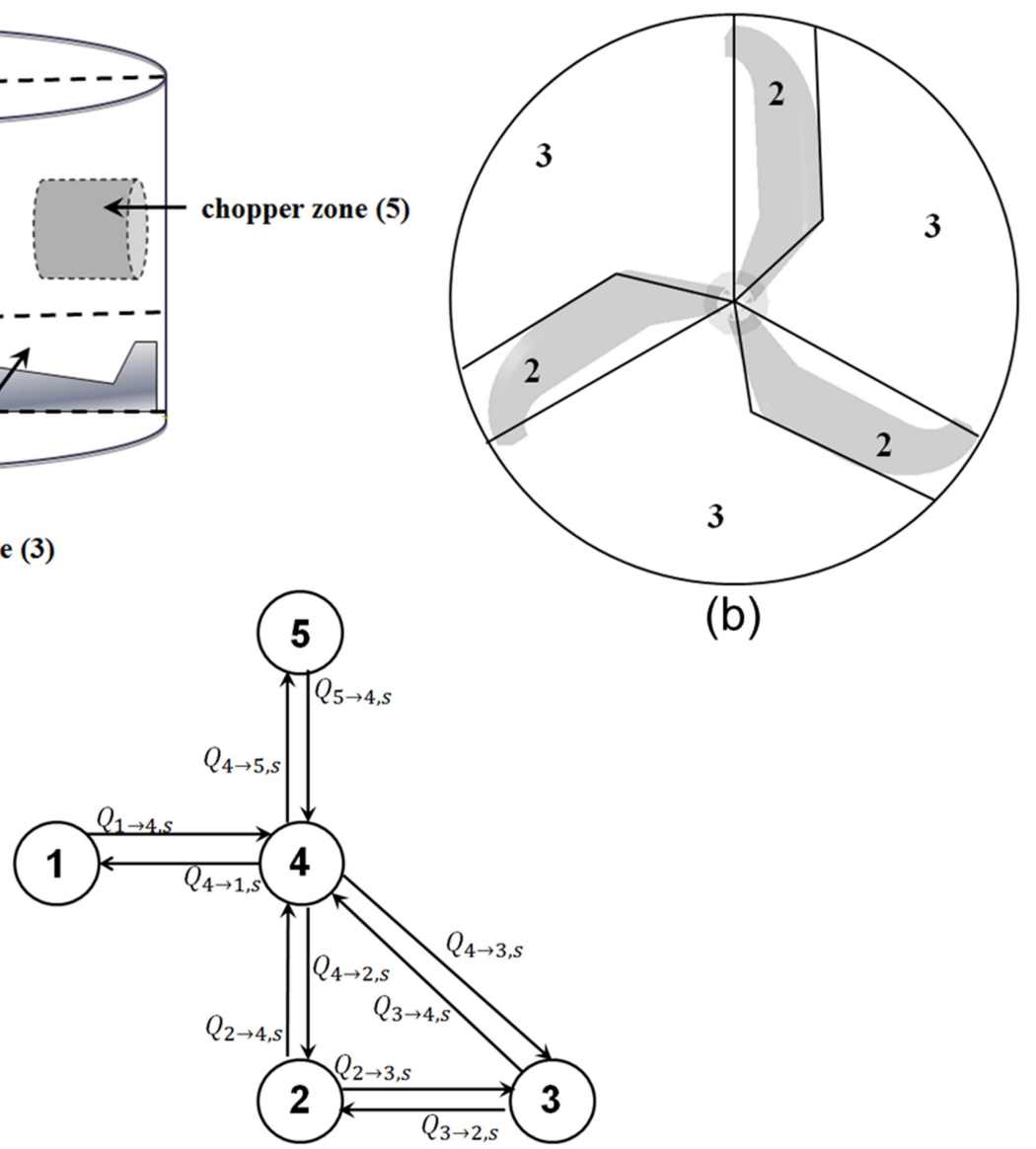

(b)

(c)

Fig 6 Compartment structure of (a) the location of compartments (b) top view of rotated compartment 2 and 3 (c) the connectivity diagram

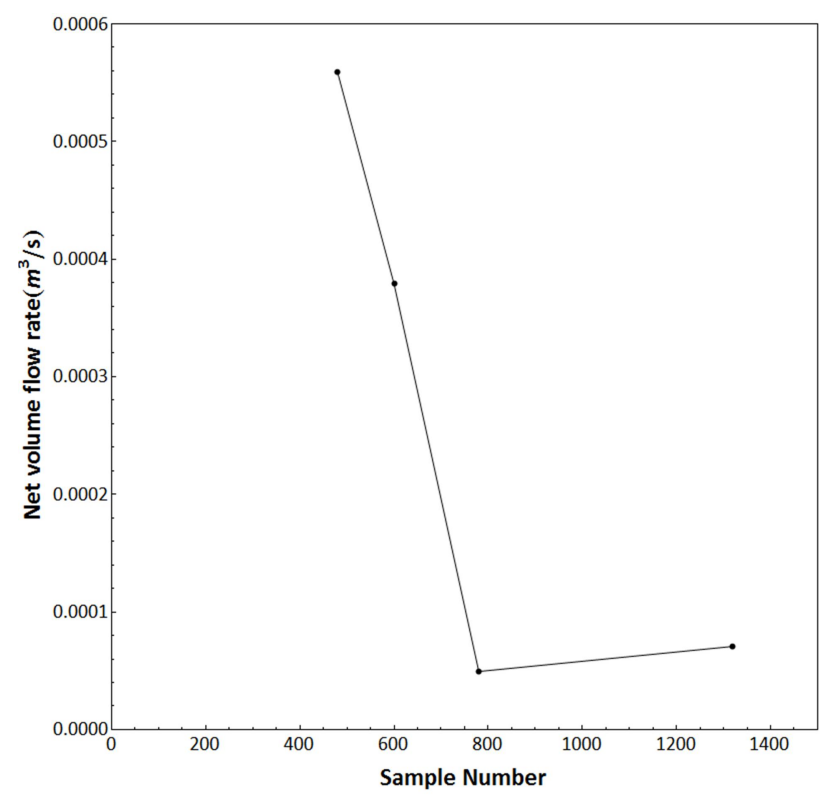

Fig 7 The impact of sample number on overall net transport rate in case II 

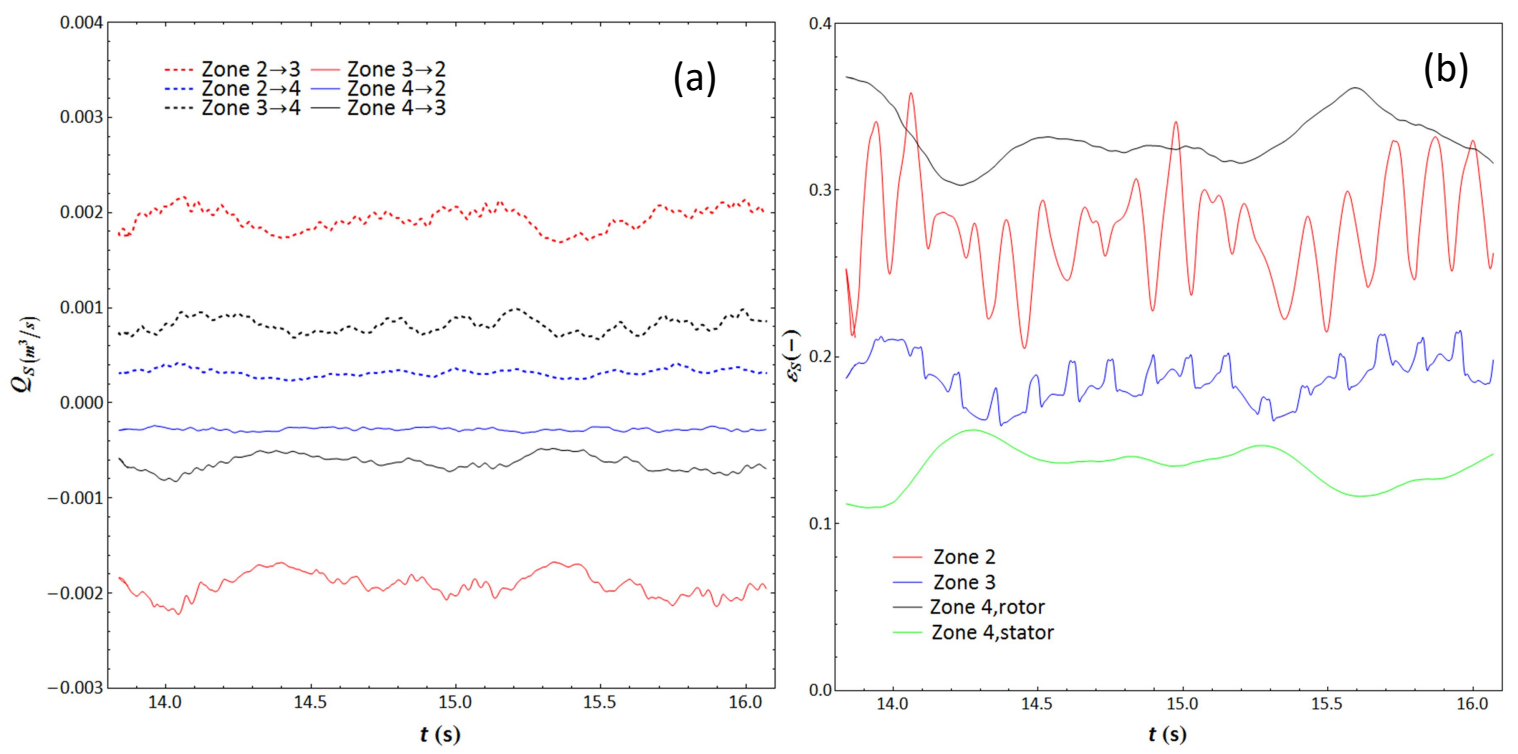

Fig 8 Temporal history of (a) volume flow rate for solid phase from compartment $i$ to $j$ in case I, 2 represents Impeller zone 2; 3 represents Bulk zone 3; 4 represents Bulk zone 4. (b) solid volume fraction in Impeller zone 2, Bulk zone 3, rotor part of Bulk zone 4 and stator part of Bulk zone 4 in case I 

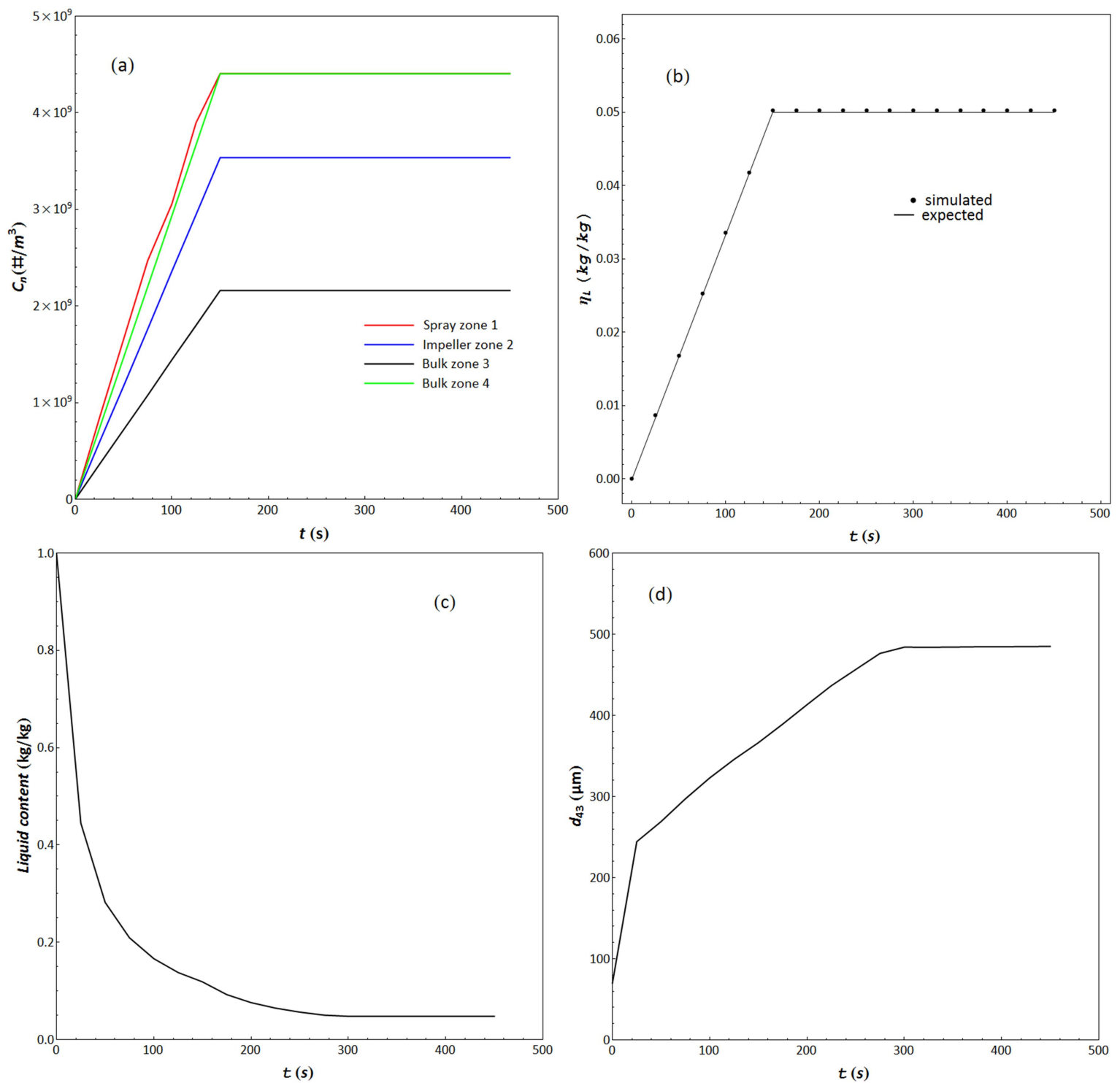

Fig 9: Temporal evolution of (a) predicted number concentration of granules for four compartments $(b)$ the expected $(-)$ and simulated $(\bullet)$ mass ratio $\eta_{L}(c)$ predicted overall liquid content within granules (d) predicted of granule mean size $d_{43}$ using test 7 in case $I$ 


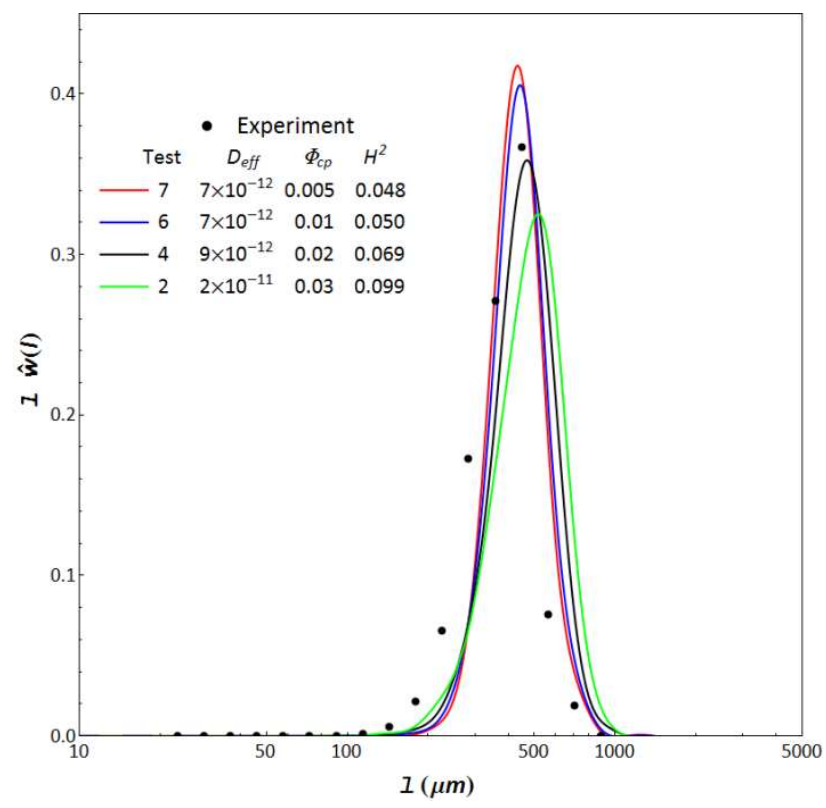

Fig 10: Comparison between the simulated $(-)$ and measured $(\bullet)$ granule size distribution (GSD) at 450s at $\Phi_{C P}=0.005-0.03$ in case I

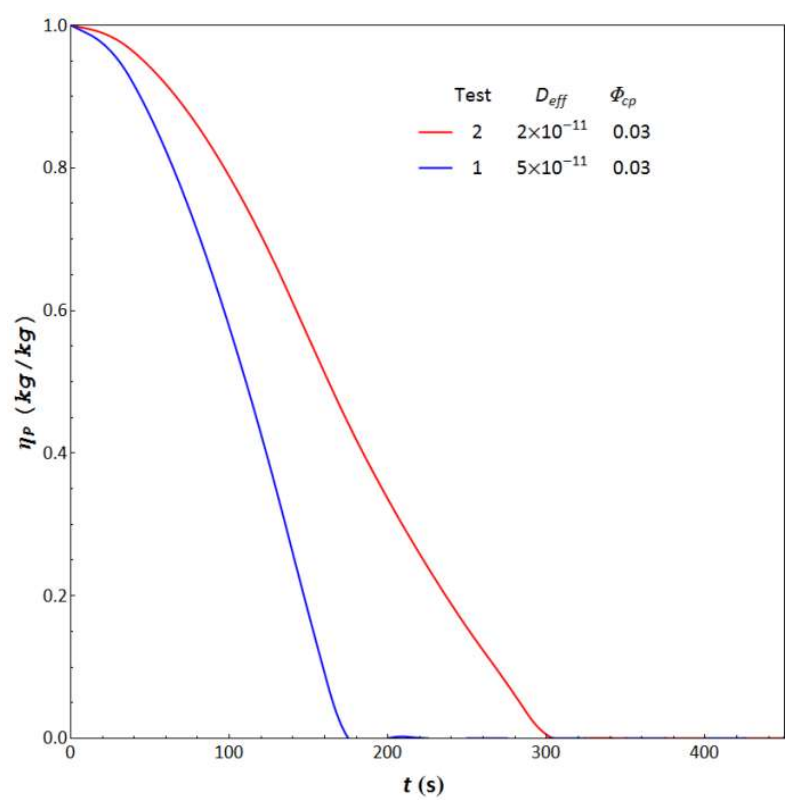

Fig 11: Predicted mass ratio $\eta_{P}$ against time at 450s using test 2 and 1 in case I 

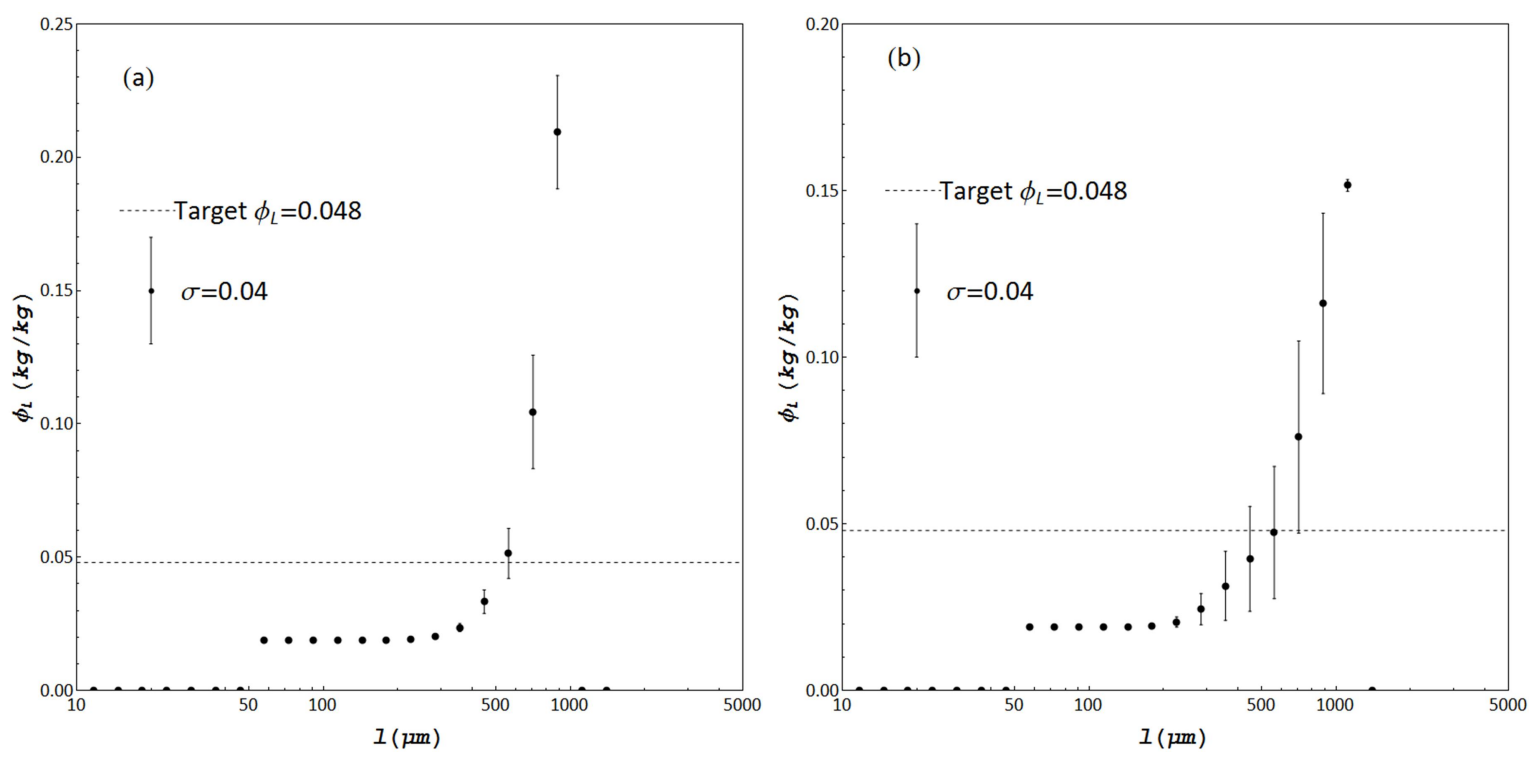

Fig 12: Predicted mean liquid content and its standard deviation against granule diameter at 450s using (a) $D_{\text {eff }}=2 \times 10^{-11}$, test 2 (b) $D_{\text {eff }}=5 \times 10^{-11}$, test 1 ; the error bar indicates the standard deviation of liquid mass fraction at each granule diameter; the target liquid mass fraction in these graphs with a dashed line in case I

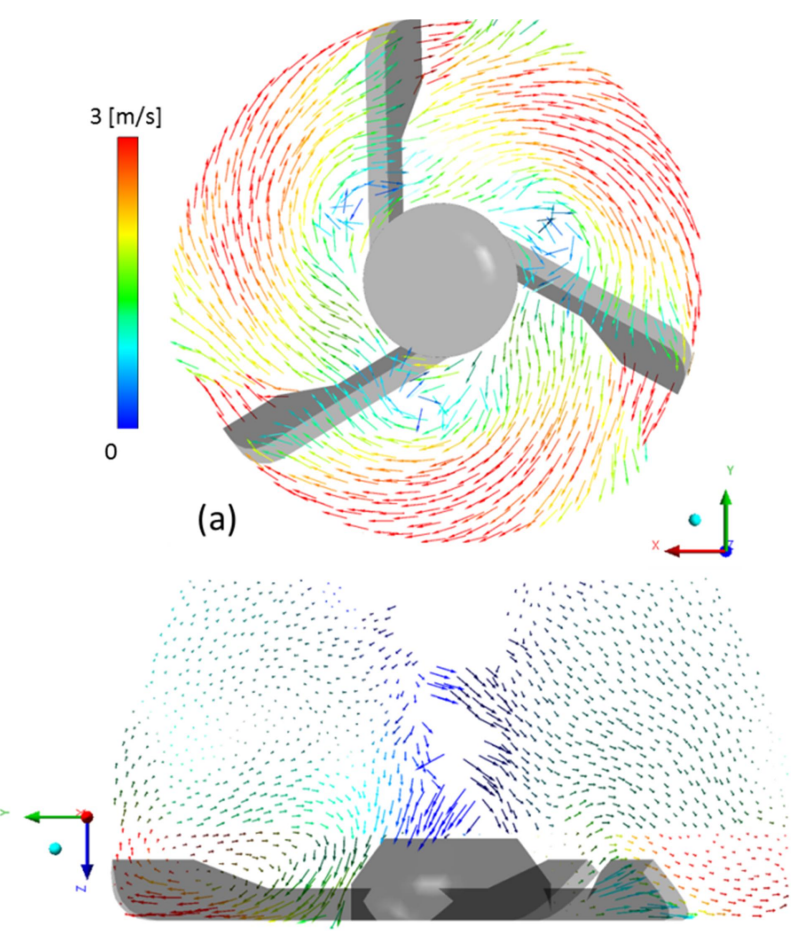

(b)

Fig 13 Velocity vector for solid phase (a) top view $(z=10 H / 113)$ (b) cross-section $(x=0)$ at steady condition in case II 

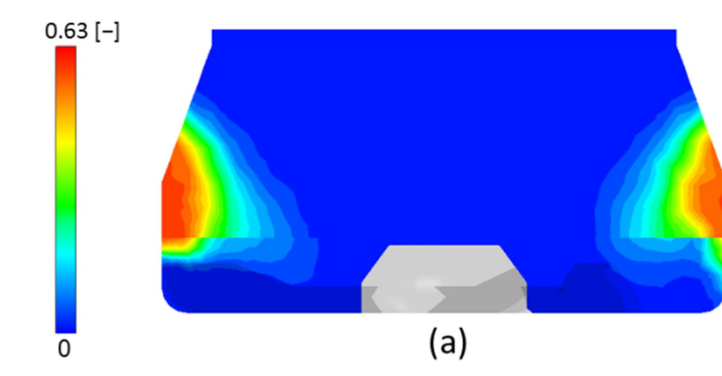

(a)
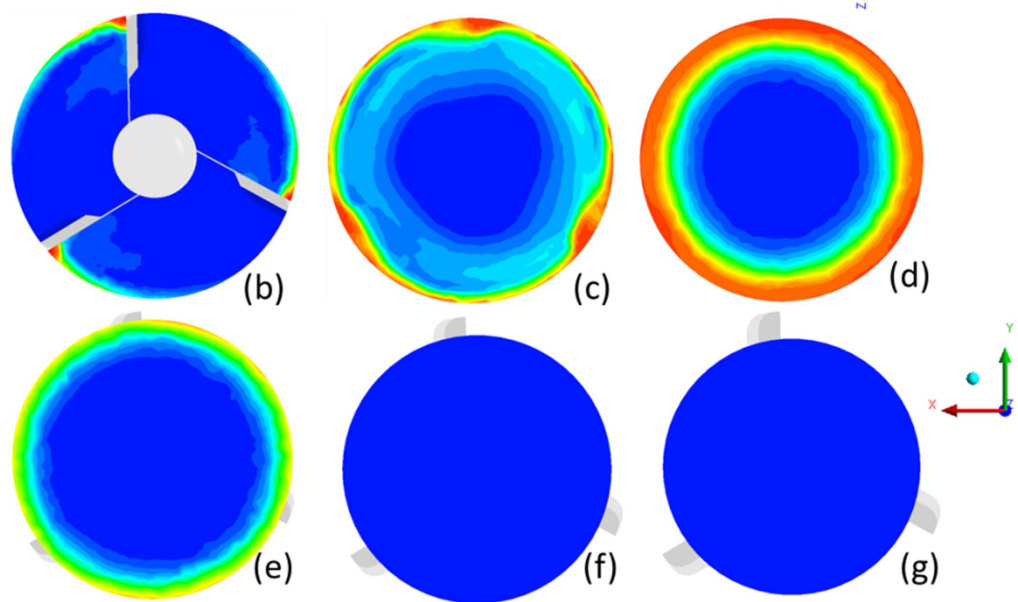

Fig 14 Countor plot of solid concentration profile (a) cross-section of $x=0$, and top view of (b) $z=10 \mathrm{H} / 113$ (c) $z=30 \mathrm{H} / 113$ (d) $z=50 \mathrm{H} / 113$ (e) $z=70 \mathrm{H} / 113$ (f) $z=90 \mathrm{H} / 113$ (g) $z=110 \mathrm{H} / 113$ at steady condition in case II
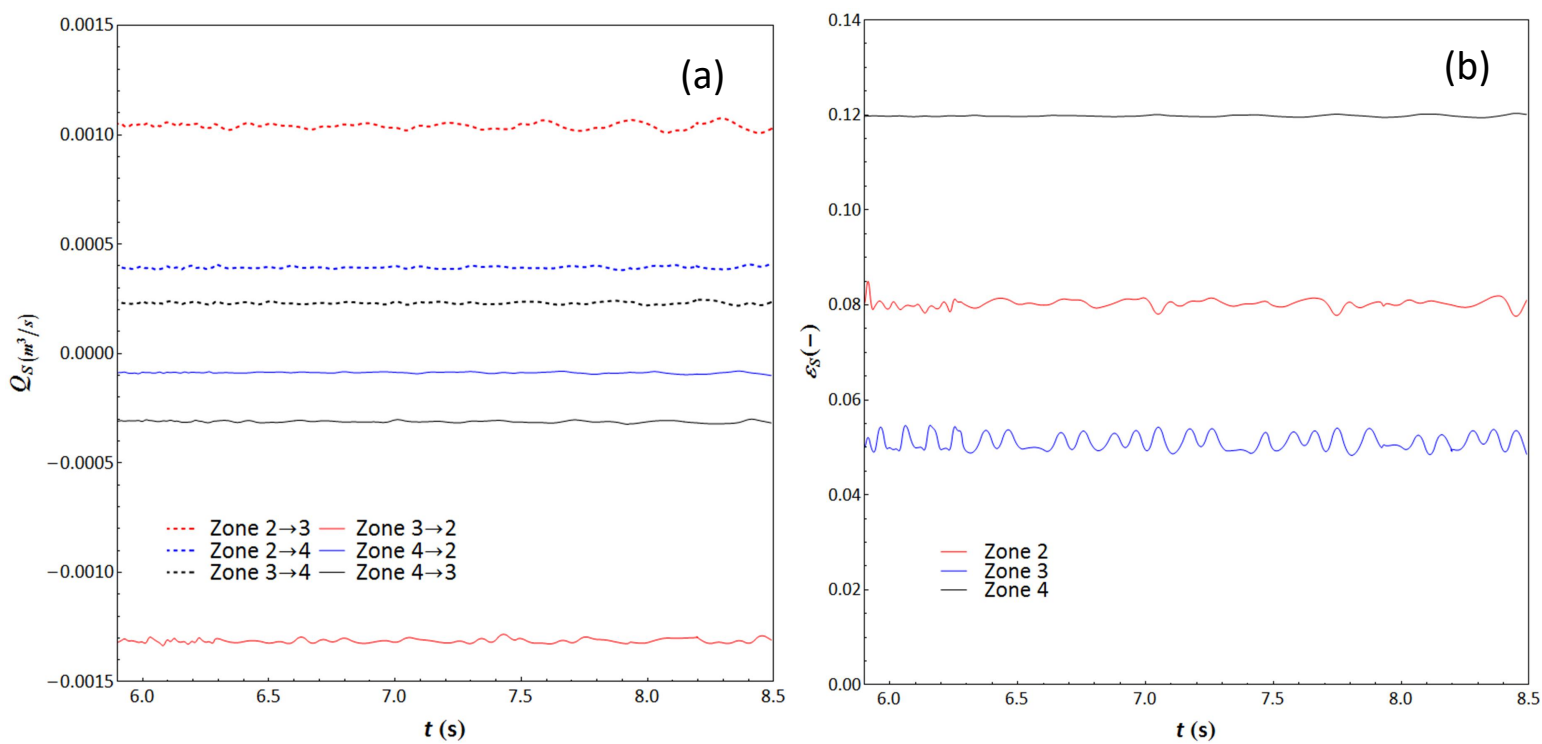

Fig 15 Temporal history of (a) volume flow rate for solid phase from compartment $i$ to $j$ in case II, (2 represents Impeller zone 2; 3 represents Bulk zone 3; 4 represents Bulk zone 4). (b) solid volume fraction in Impeller zone 2, Bulk zone 3 and Bulk zone 4 for case II 

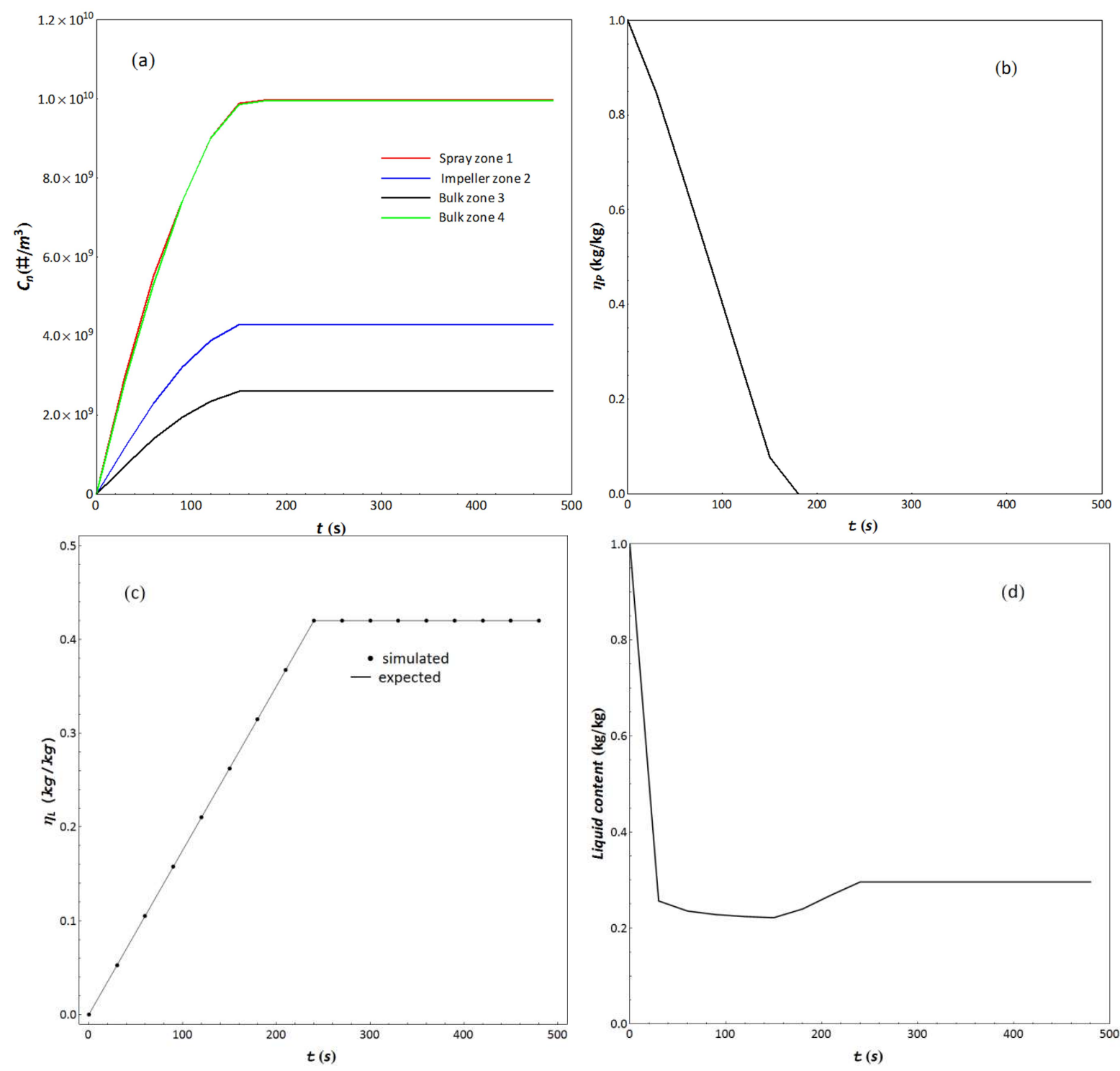

Fig 16: Temporal evolution of (a) predicted number concentration of granules for four compartments (b) predicted mass ratio $\eta_{P}(c)$ the expected $(-)$ and simulated $(\bullet)$ mass ratio $\eta_{L}(d)$ predicted overall liquid content within granules using test 1 in case II. 

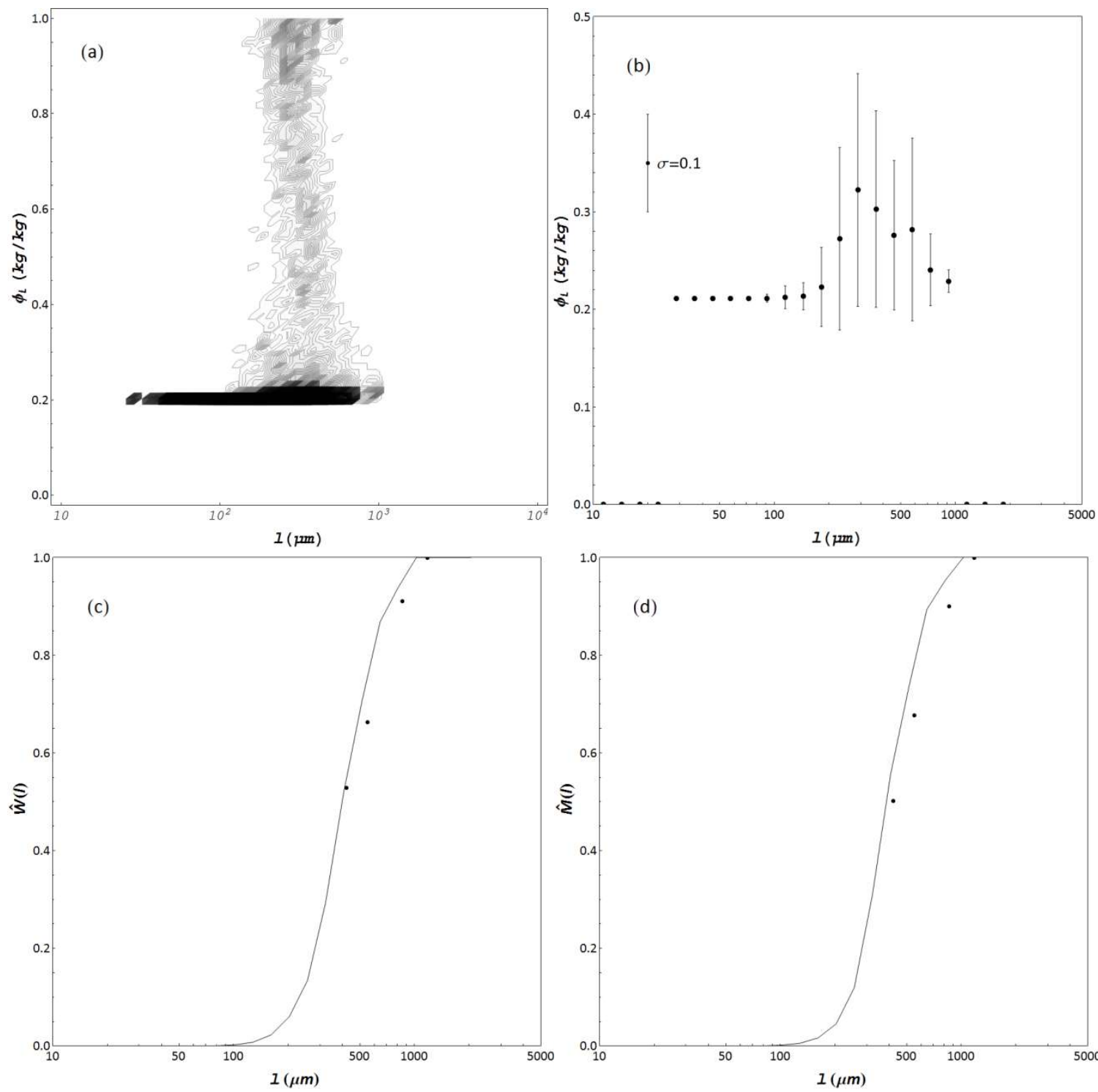

Fig 17: (a) predicted liquid content(liquid mass fraction) distribution against granule size (contour plot) [The weight of the contour shading indicates relative number density.] (b) predicted mean liquid mass fraction and standard deviation against granule diameter [The error bar indicates the standard deviation of liquid mass fraction at each granule diameter] (c) comparison the simulated (-) and measured $(\bullet)$ cumulative GSD (d) comparison the simulated $(-)$ and measured $(\bullet)$ cumulative BSD at 240s using test 1 in case II. 


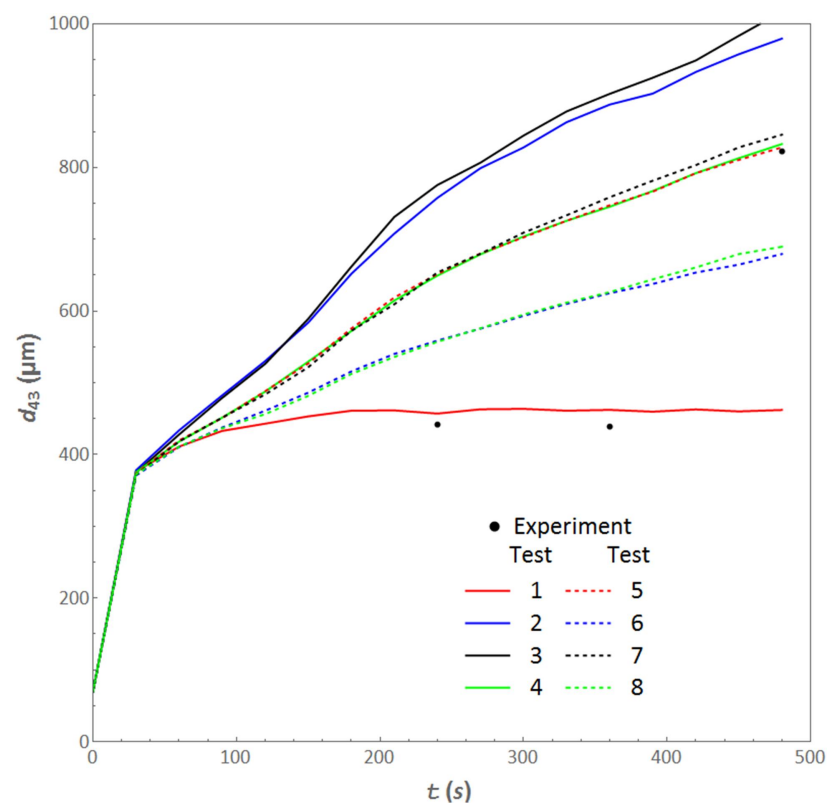

Fig 18: Comparison between the simulated $(-)$ and measured $(\bullet)$ granule mean size $d_{43}$ against time in case II.
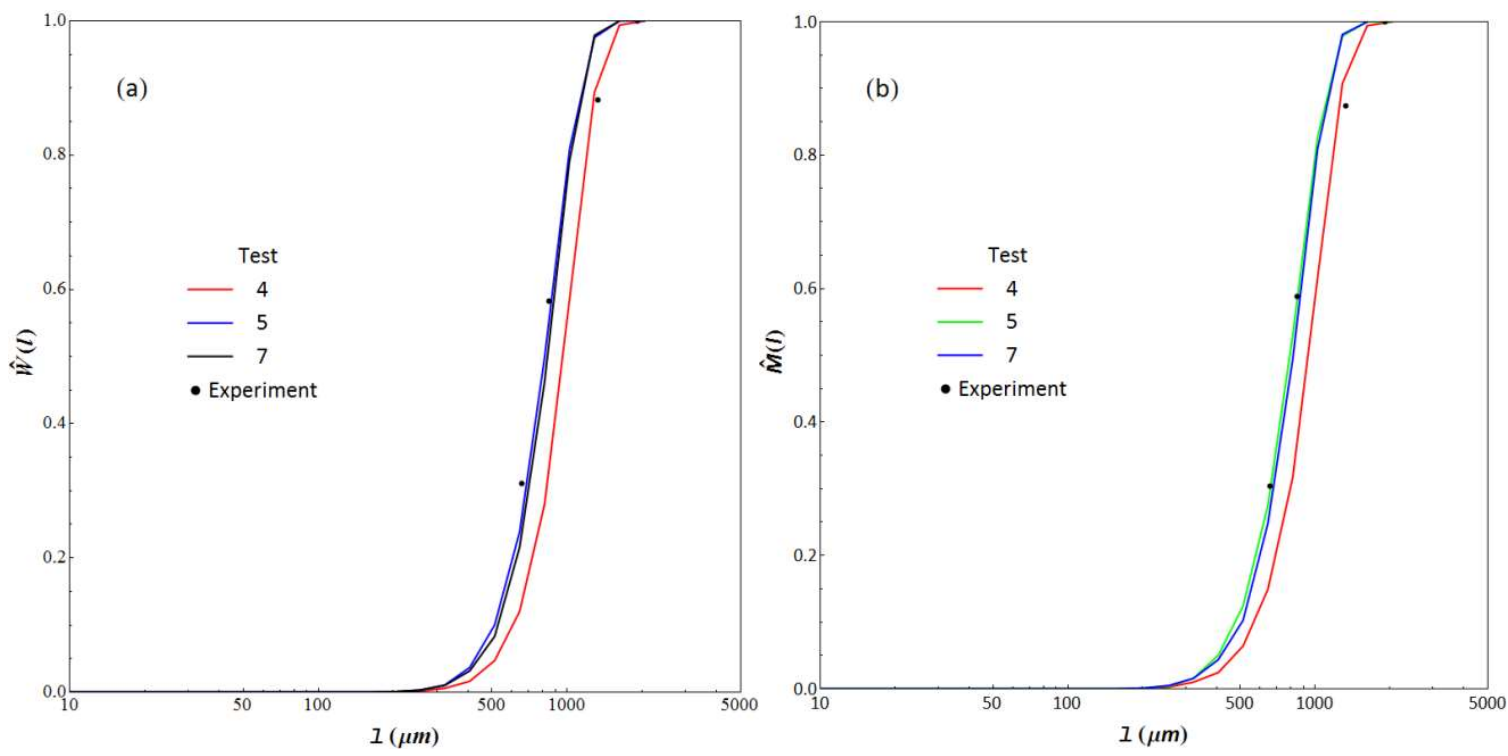

Fig 19: Comparison between the simulated $(-)$ and measured $(\bullet)$ cumulative probability distribution based on (a) GSD (b) BSD at 480s using test 4, 5 and 7 in case II. 